\title{
Cyanoacetylene in the outflow/hot molecular core G331.512-0.103
}

\author{
N. U. Duronea, ${ }^{1 \star}$, L. Bronfman ${ }^{2}$, E. Mendoza ${ }^{3}$, M. Merello ${ }^{3}$, R. Finger ${ }^{2}$, \\ N. Reyes ${ }^{2,4}$, C. Hervías-Caimapo ${ }^{5}$, A. Faure ${ }^{6}$, C.E. Cappa ${ }^{7}$, E. M. Arnal ${ }^{7}$, \\ J.R.D. Lépine ${ }^{3}$, I. Kleiner ${ }^{8}$, and L-A. Nyman ${ }^{9}$ \\ ${ }^{1}$ Instituto Argentino de Radioastronomía, CONICET, CCT-La Plata, C.C.5., 1894, Villa Elisa, and CIC, Prov. de Bs. As. Argentina \\ ${ }^{2}$ Departamento de Astronomía, Universidad de Chile, Casilla 36, Santiago de Chile \\ ${ }^{3}$ Instituto de Astronomia, Geofísica e Ciências Atmosféricas, Universidad de São Pãulo, São Pãulo 05508-090, SP, Brazil \\ ${ }^{4}$ Departamento de Ingeniería Eléctrica, Universidad de Chile, Santiago de Chile. \\ ${ }^{5}$ Department of Physics, Florida State University, Tallahassee, FL 32306, USA \\ ${ }^{6}$ Université Grenoble Alpes, CNRS, IPAG, 38000 Grenoble, France \\ ${ }^{7}$ Facultad de Ciencias Astronómicas y Geofísicas, Universidad Nacional de La Plata, Paseo del Bosque s/n, 1900 La Plata, Argentina \\ ${ }^{8}$ Laboratoire Interuniversitaire des Systemes Atmospheriques (LISA), CNRS, UMR 7583, Université de Paris-Est Créteil et Paris Diderot, \\ 61, Av Général de Gaulle, 94010 Créteil Cedex, France \\ ${ }^{9}$ Joint ALMA Observatory, JAO Alonso de Córdova 31070, Vitacura, Santiago de Chile, Chile.
}

Accepted XXX. Received YYY; in original form ZZZ

\begin{abstract}
Using APEX-1 and APEX-2 observations, we have detected and studied the rotational lines of the $\mathrm{HC}_{3} \mathrm{~N}$ molecule (cyanoacetylene) in the powerful outflow/hot molecular core G331.512-0.103. We identified thirty-one rotational lines at $J$ levels between 24 and 39; seventeen of them in the ground vibrational state $v=0 \quad(9$ lines corresponding to the main $\mathrm{C}$ isotopologue and 8 lines corresponding to the ${ }^{13} \mathrm{C}$ isotopologues), and fourteen in the lowest vibrationally excited state $v_{7}=1$. Using LTE-based population diagrams for the beam-diluted $v=0$ transitions, we determined $T_{\text {exc }}=85 \pm 4 \mathrm{~K}$ and $N\left(\mathrm{HC}_{3} \mathrm{~N}\right)=(6.9 \pm 0.8) \times 10^{14} \mathrm{~cm}^{-2}$, while for the beam-diluted $v_{7}=1$ transitions we obtained $T_{\text {exc }}=89 \pm 10 \mathrm{~K}$ and $N\left(\mathrm{HC}_{3} \mathrm{~N}\right)=2 \pm 1 \times 10^{15} \mathrm{~cm}^{-2}$. Non-LTE calculations using $\mathrm{H}_{2}$ collision rates indicate that the $\mathrm{HC}_{3} \mathrm{~N}$ emission is in good agreement with LTE-based results. From the non-LTE method we estimated $T_{\text {kin }} \simeq 90 \mathrm{~K}$, $n\left(\mathrm{H}_{2}\right) \simeq 2 \times 10^{7} \mathrm{~cm}^{-3}$ for a central core of 6 arcsec in size. A vibrational temperature in the range from $130 \mathrm{~K}$ to $145 \mathrm{~K}$ was also determined, values which are very likely lower limits. Our results suggest that rotational transitions are thermalized, while IR radiative pumping processes are probably more efficient than collisions in exciting the molecule to the vibrationally excited state $v_{7}=1$. Abundance ratios derived under LTE conditions for the ${ }^{13} \mathrm{C}$ isotopologues suggest that the main formation pathway of $\mathrm{HC}_{3} \mathrm{~N}$ is $\mathrm{C}_{2} \mathrm{H}_{2}+\mathrm{CN} \rightarrow \mathrm{HC}_{3} \mathrm{~N}+\mathrm{H}$.
\end{abstract}

Key words: ISM: jets and outflows - ISM: astrochemistry - stars: formation

\section{INTRODUCTION}

Cyanopolyynes molecules $\left(\mathrm{HC}_{2 n+1} \mathrm{~N}, n \geqslant 1\right)$ are typical carbon-chain molecules that seem to be ubiquitous in the interstellar medium (ISM) since they have been detected in a wide range of environments both in the Milky Way and in other galaxies. The shortest member of the family, $\mathrm{HC}_{3} \mathrm{~N}$ (cyanoacetylene; $\mathrm{H}-\mathrm{C} \equiv \mathrm{C}-\mathrm{C} \equiv \mathrm{N}$ ), is the most abun-

* E-mail: duronea@iar.unlp.edu.ar dant cyanopolyyne. Due to its low rotational constant (1/13 of CO) and large dipole moment (3.7 D, DeLeon \& Muenter 1985), cyanoacetylene lines can be detected over a wide range of rotational lines (closely spaced by only $9.1 \mathrm{GHz}$ ) at relatively low frequencies, in contrast with, for example, $\mathrm{HCN}$ or $\mathrm{HCO}^{+}$, which have high- $J$ lines at very high frequencies that are extremely difficult to detect with groundbased telescopes. The critical density of $\mathrm{HC}_{3} \mathrm{~N}\left(n_{\mathrm{H}_{2}} \sim 10^{5}\right.$ $\left.10^{6} \mathrm{~cm}^{-3}\right)$ is comparable to other high density gas tracers, such as $\mathrm{HCN}, \mathrm{CS}$, or $\mathrm{HCO}^{+}$. In addition, $\mathrm{HC}_{3} \mathrm{~N}$ is usually 


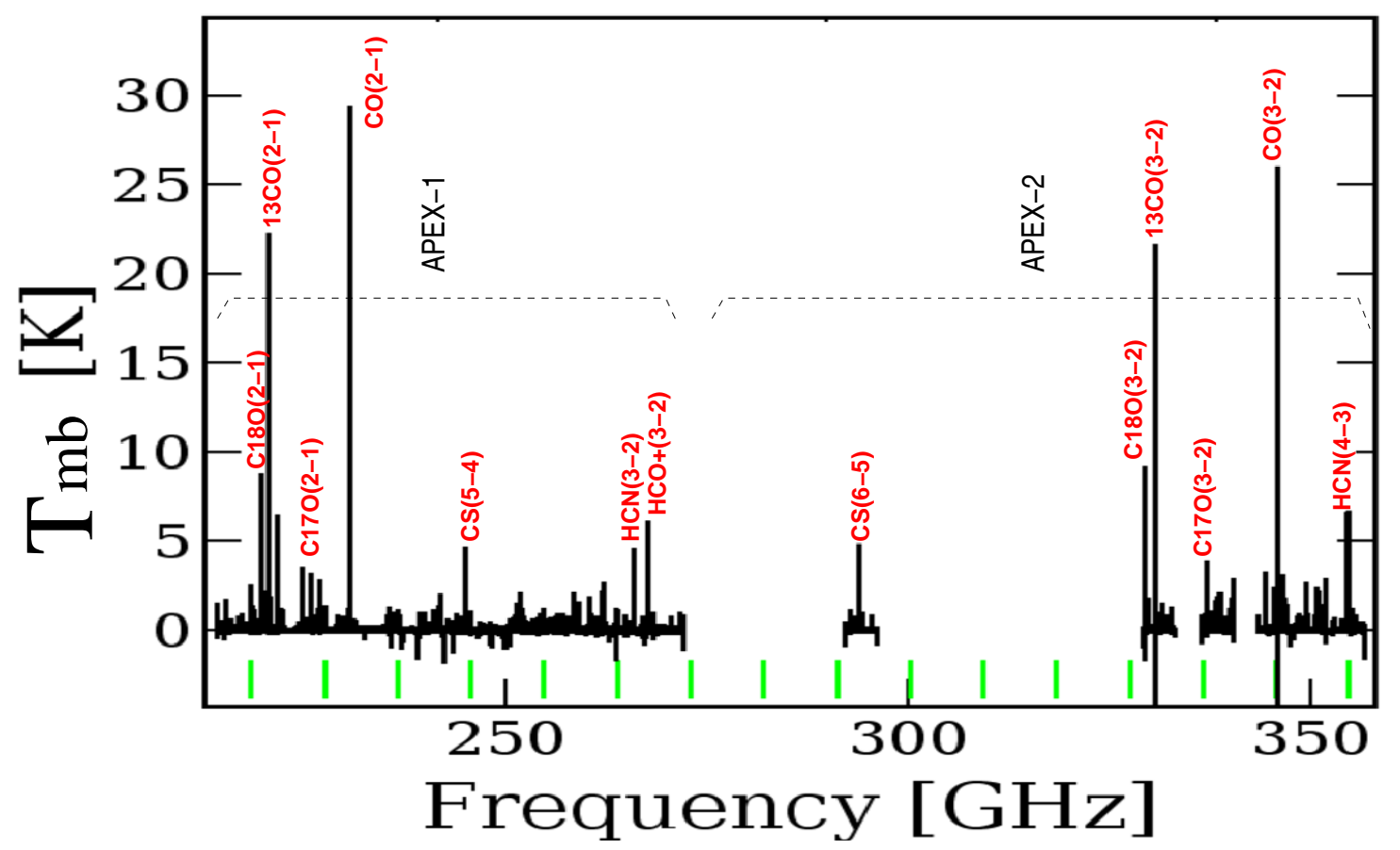

Figure 1. Total spectrum obtained with APEX-1 and APEX-2 data, used to identify the $\mathrm{HC}_{3} \mathrm{~N}$ lines. The rest frequencies of the $v=0$ transitions of the main $\mathrm{C}$ isotopologue of $\mathrm{HC}_{3} \mathrm{~N}$ are indicated by the green dashes at the bottom of the spectrum. Some of the most prominent and characteristic molecular transitions in the observed bands are indicated in red as a reference.

optically thin even in low- $J$ transitions, mostly due to its relatively low abundance, which is suitable for studying dense molecular gas (e.g Bergin et al. 1996). This makes it easier to conduct multi-transition analysis for $\mathrm{HC}_{3} \mathrm{~N}$ than for other dense molecular gas tracers, which can help to better understand the physical and excitation conditions of dense molecular gas in star forming regions. Further, the $\mathrm{HC}_{3} \mathrm{~N}$ molecule can be excited not only in the ground vibrational state, but also in several vibrationally excited states that can also be used to derive important information about the physical characteristics of the region under study, such as kinetic and vibrational temperatures, density, etc. (Wyrowski et al. 1999; Martín-Pintado et al. 2005; Jiménez-Serra et al. 2009; Peng et al. 2017).

Several lines of $\mathrm{HC}_{3} \mathrm{~N}$ have been widely detected in a number of hot cores and high-mass star forming regions (Bergin et al. 1996; Kim et al. 2000; de Vicente et al. 2000; Sollins \& Megeath 2004; Belloche et al. 2013; Sánchez-Monge et al. 2013; Taniguchi et al. 2016b, 2018b,c). Surveys of sources at different evolutionary stages, from high-mass starless cores to protostellar objects, reveal the presence of spectral signatures of both $\mathrm{HC}_{3} \mathrm{~N}$ and $\mathrm{HC}_{5} \mathrm{~N}$ (Taniguchi et al. 2018a). In the protostellar shock L1157-B1, including observations toward its protostar L1157-mm, lines of $\mathrm{HC}_{3} \mathrm{~N}$ and $\mathrm{HC}_{5} \mathrm{~N}$ were observed showing different physical components of the gas (Mendoza et al. 2018a). In the solar-type protostar IRAS 16293-2422, observations of $\mathrm{HC}_{3} \mathrm{~N}$ and $\mathrm{HC}_{5} \mathrm{~N}$ also revealed different gas conditions between the outer and inner regions of the protostellar system (Jaber Al-Edhari et al. 2017). In protoplanetary disks, observations at high spatial resolution of $\mathrm{HC}_{3} \mathrm{~N}$ and $\mathrm{CH}_{3} \mathrm{CN}$ show abundance ratios of those molecules in agreement with protostellar envelopes and comets (Bergner et al. 2018).

As part of a project aimed at studying in detail the physical and chemical properties of the massive outflow/hot molecular core G331.512-0.103 (Bronfman et al. 2008), in this paper we present an analysis of the above source based on APEX observations of $\mathrm{HC}_{3} \mathrm{~N}$ lines. The excellent spectral and spatial resolution of the SHeFI APEX-1 and APEX-2 data, along with the obtained spectral coverage, provides a powerful tool to perform a complete analysis on the source.

\section{THE MOLECULAR OUTFLOW/HOT MOLECULAR CORE G331.512-0.103}

G331.512-0.103 (hereafter G331) is a bright massive young stellar object associated with the central region of the giant molecular cloud (GMC) G331.5-0.1 (Bronfman et al. 2008; Merello et al. 2013a), which is located at the tangent point of the Norma spiral arm at a heliocentric distance of $\sim 7.5 \mathrm{kpc}$. The presence of very broad emission wings in $\mathrm{CO}, \mathrm{CS}$, and $\mathrm{SiO}$ indicates a very powerful outflow with a mass of around $55 M \odot$ and a momentum of $\sim 2.4 \times 10^{3}$ $M_{\odot} \mathrm{km} \mathrm{s}^{-1}$ (Bronfman et al. 2008). Merello et al. (2013b) analyzed ALMA $\mathrm{SiO}(8-7), \mathrm{H}^{13} \mathrm{CO}^{+}(4-3), \mathrm{HCO}^{+}(4-3)$, and $\mathrm{CO}(3-2)$ observations towards G331 revealing the existence of lobes closely aligned with the line of sight and an expanding bubble blown by stellar winds likely arising from a hyper compact HII region confined within an angular extent of about $5 \operatorname{arcsec}(\sim 0.18 \mathrm{pc}$ at a distance of $7.5 \mathrm{kpc})$.

Several transitions of $\mathrm{CH}_{3} \mathrm{CN}$ and $\mathrm{CH}_{3} \mathrm{OH}$ have also been reported in the central core harbouring G331 (Mendoza et al. 2018b). The results revealed that the emis- 
sion of $\mathrm{CH}_{3} \mathrm{CN}$ and $\mathrm{CH}_{3} \mathrm{OH}$ are related to gas components having kinetic temperatures of $\mathrm{T}_{\mathrm{k}} \sim 141 \mathrm{~K}$ and $\mathrm{T}_{\mathrm{k}} \sim 74 \mathrm{~K}$, respectively. In agreement with previous high-resolution interferometric observations, the models show that the emitting region is compact ( $\leqslant 5.5$ arcsec), with a gas volume density $n_{\mathrm{H}_{2}} \simeq 1 \times 10^{7} \mathrm{~cm}^{-3}$. Recently, ALMA observations have revealed the presence of $\mathrm{HC}_{3} \mathrm{~N} \quad J=38-37$ rotational line (Hervías-Caimapo et al. 2019). The authors identified two group of lines. The first group, showing broad $(\sim 20$ $\mathrm{km} \mathrm{s}^{-1}$ ) velocity wings, includes $\mathrm{HC}_{3} \mathrm{~N}, \mathrm{SiO}, \mathrm{S}^{18} \mathrm{O}, \mathrm{HCO}^{+}$, and $\mathrm{H}^{13} \mathrm{CN}$; very likely they trace the outflow and shocked gas emission. The second group, with narrow $\left(6-10 \mathrm{~km} \mathrm{~s}^{-1}\right)$ emission lines, includes $\mathrm{CH}_{3} \mathrm{CCH}, \mathrm{CH}_{3} \mathrm{OH}$ and $\mathrm{H}^{13} \mathrm{CO}^{+}$ lines. They may be arising from the quiescent core ambient medium.

The physical, chemical, and kinematical properties of G331 make this object one of the most massive, powerful, and youngest hot cores/outflows known to date.

\section{OBSERVATIONS, DATABASES, AND METHODOLOGY}

The molecular observations presented in this paper were obtained with the 12-m APEX telescope (Güsten et al. 2006) at Llano de Chajnantor (Chilean Andes) in May 2017. As front end for the observations we used the APEX-1 receiver of the Swedish Heterodyne Facility Instrument (SHeFI; Risacher et al. 2006; Vassilev et al. 2008). The back end for the observations was the eXtended bandwidth Fast Fourier Transform Spectrometer2 (XFFTS2) which consists of two units with $2.5 \mathrm{GHz}$ bandwidth divided into 32768 channels each. The half power beam width (HPBW) in the APEX-1 frequency band varies between $25-30$ arcsec. Calibration was performed using the chopper-wheel technique. The output intensity scale given by the system is $T_{\mathrm{A}}^{*}$, which represents the antenna temperature corrected for atmospheric attenuation. The observed intensities were converted to mainbeam brightness temperature by $T_{\mathrm{mb}}=T_{\mathrm{A}}^{*} / \eta_{\mathrm{mb}}(\lambda)$, where $\eta_{\mathrm{mb}}(\lambda)$ was obtained using the Ruzes's equation for a mean main beam efficiency of 0.75 and a surface accuracy (rms) of $17 \mu \mathrm{m}$. The spectra were obtained in the single point mode in the coordinates RA, Dec. $(\mathrm{J} 2000)=16^{h} 12^{m} 10.1^{s}$, $-51^{\circ} 28^{\prime} 38.1^{\prime \prime}$ in sixteen frequency setups to cover the entire APEX-1 band (214 to $272 \mathrm{GHz}$ ). Because only up to $4 \mathrm{GHz}$ total instantaneous bandwidth can be used, frequency setups were spaced by $3.7 \mathrm{GHz}$ in order to avoid noisy marginal detections. The spectra were hanning-smoothed to obtain a final rms noise of $\sim 0.025-0.05 \mathrm{~K}$ and a final spectral resolution of $\sim 0.4 \mathrm{~km} \mathrm{~s}^{-1}$ for all the setups.

The spectra were reduced using the CLASS90 program of the IRAM GILDAS software package ${ }^{1}$. The analysis of the spectra and the line identification was performed using the CASSIS software ${ }^{2}$ conjointly with the $\mathrm{CDMS}^{3}$ (Müller et al. 2005), $\mathrm{JPL}^{4}$ (Pickett et al. 1998), and $\mathrm{NIST}^{5}$ (Lovas et al. 2009) spectroscopic databases.

\footnotetext{
1 http://www.iram.fr/IRAMFR/GILDAS

2 http://cassis.irap.omp.eu

3 https://www.astro.uni-koeln.de/cdms

4 https://spec.jpl.nasa.gov/

5 https://physics.nist.gov/cgi-bin/micro/table5/start.pl
}

The APEX-1 observations used in this work were combined with previous observations carried out using the APEX-2 instrument (HPBW $=17-23$ arcsec) (see Fig. 1). We adopted calibration uncertainties of around 20 percent (Dumke \& Mac-Auliffe 2010).

\section{RESULTS AND ANALYSIS}

\subsection{Line identification}

The APEX-1 and APEX-2 data used in this work cover the total frequency range between $214 \mathrm{GHz}$ and $356.5 \mathrm{GHz}$, with the APEX-1 frequency range between 214 and 272 fully covered and some gaps in the APEX-2 band between 272 and $292 \mathrm{GHz}, 296$ and $329 \mathrm{GHz}, 333$ and 336.4, and 340.4 and $343.3 \mathrm{GHz}$ (see Fig. 1). Nevertheless, the covered frequency range allows the detection of a considerable number of $\mathrm{HC}_{3} \mathrm{~N}$ lines, having an upper level energy $\left(E_{u}\right)$ of the main isotopologue between $\sim 131 \mathrm{~K}$ and $\sim 341 \mathrm{~K}$. The detection of these lines is necessary to perform a high accuracy determination of some physical parameters in the region (e.g. column density, kinetic temperature, opacity, etc.).

Since the central region of G331 shows a very rich hot molecular core chemistry, a forest of molecular transitions leads to a confusing spectra (see Fig. 1), especially for the presence of broad lines (Mendoza et al. 2018b; Hervías-Caimapo et al. 2019). Then, we performed the line identification adopting the following standard criteria:

(i) The observed frequencies of the lines should agree with the rest frequencies for the systemic velocity of the source $\left(\mathrm{V}_{\mathrm{lsr}} \sim-90 \mathrm{~km} \mathrm{~s}^{-1}\right)^{6}$.

(ii) The peak temperature of the lines should be at least about three times the rms noise. A couple of exceptions were included where the signal-to-noise ratio is locally a bit lower than in the rest of the spectrum.

(iii) All predicted lines based on an LTE spectrum at a well-defined rotational temperature should be present in the observed spectrum approximately at their predicted relative intensities.

Since a considerable number of predicted contaminant molecular transitions, known as weeds ${ }^{7}$, can be detected at several frequencies in the $\mathrm{mm}$ and sub-mm range, we also inspected for possible contaminant emission. Such inspection was carried out using spectral catalogs and the Weeds extension of the CLASS radio astronomy software. ${ }^{8}$ Thus, we remarked three status in the line identification (see last column of Table 1): a) lines possibly blended with known interstellar species (pbw): transitions whose FWHM values derived from the Gaussian fits coincide with predicted frequencies of interstellar species detected in the ISM (in all the cases the possible contaminat species were detected in the

6 In this work radial velocities are always referred to the Local Standard of Rest (lsr)

7 Term usually adopted to refer to species with numerous spectral lines in the $\mathrm{mm} / \mathrm{sub}-\mathrm{mm}$ regime

8 https://www.iram.fr/IRAMFR/GILDAS/doc/html/weeds-html/weeds.html 

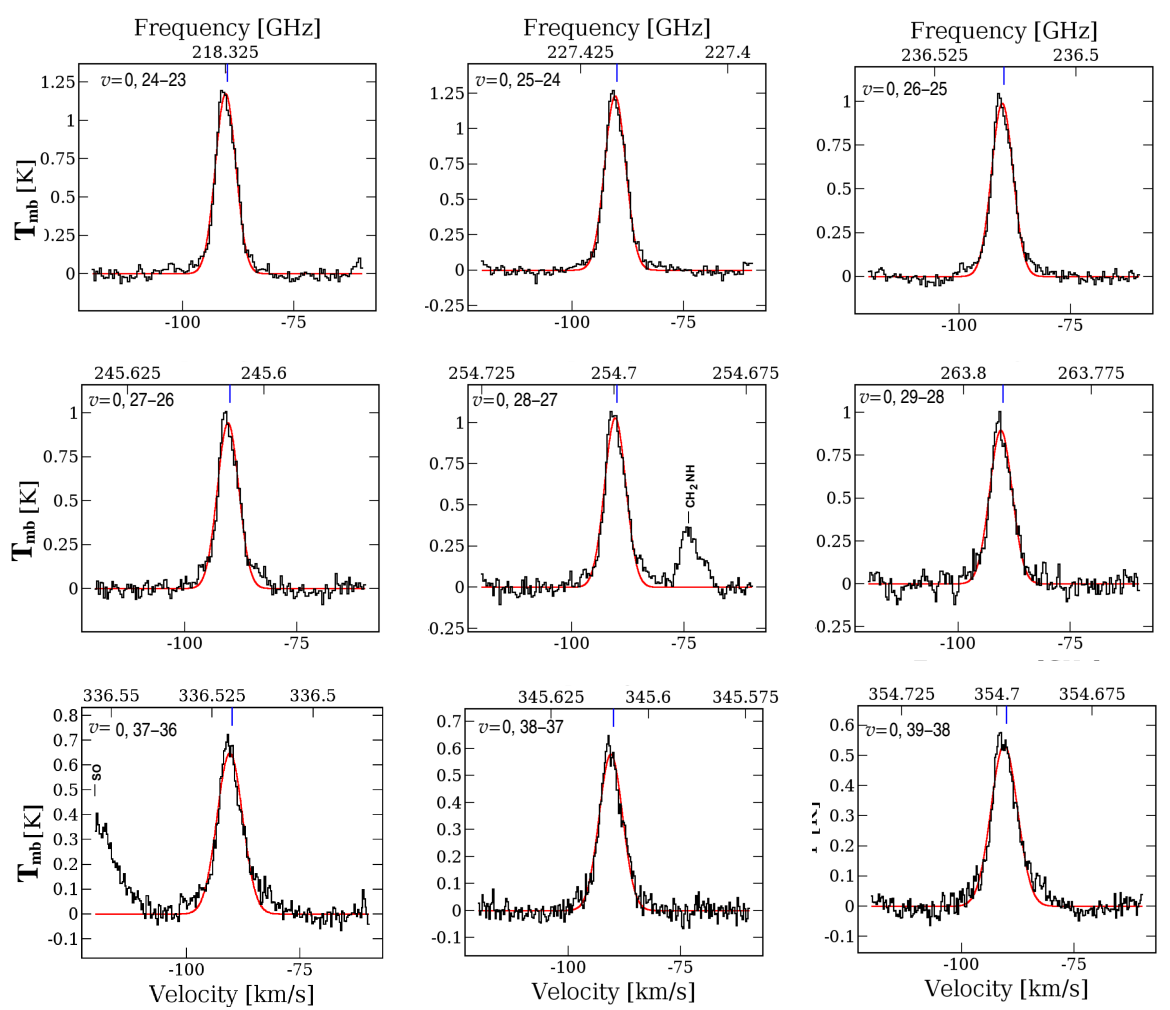

Figure 2. Observed spectra of the detected lines of $\mathrm{HC}_{3} \mathrm{~N}$ in the ground vibrational state $v=0$. The red curves show the gaussian fit to the lines. The transitions are indicated in the top left corner of each panel. The blue line at the top of the spectra indicate the rest frequency of each transition.
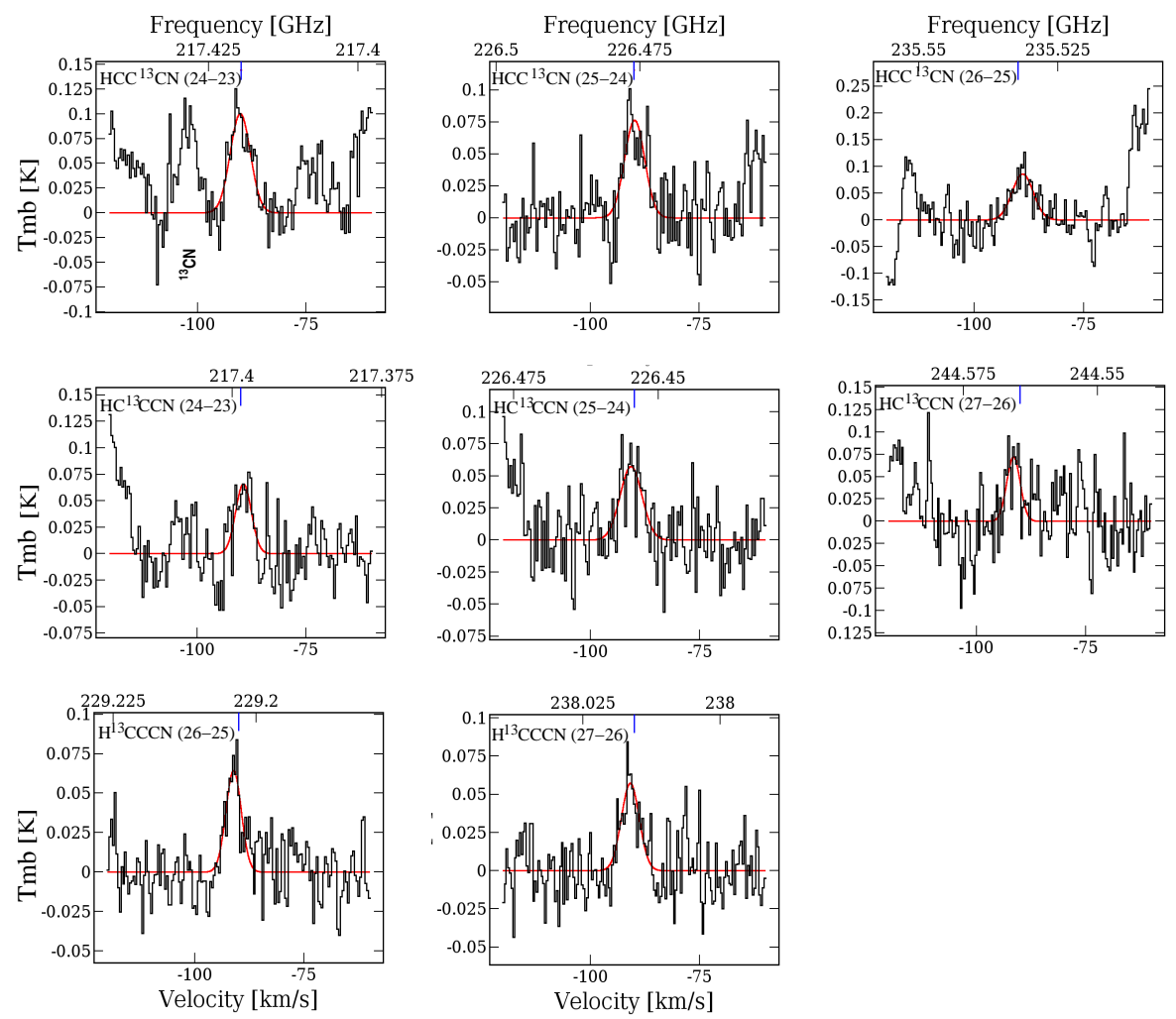

Figure 3. Observed spectra of the detected ${ }^{13} \mathrm{C}$ isotopologues of $\mathrm{HC}_{3} \mathrm{~N}$ in the ground vibrational state $v=0$. The red curves show the gaussian fit to the lines. The corresponding isotopologue and its transition are indicated in the top left corner of each panel. The blue line at the top of the spectra indicate the rest frequency of each transition. 

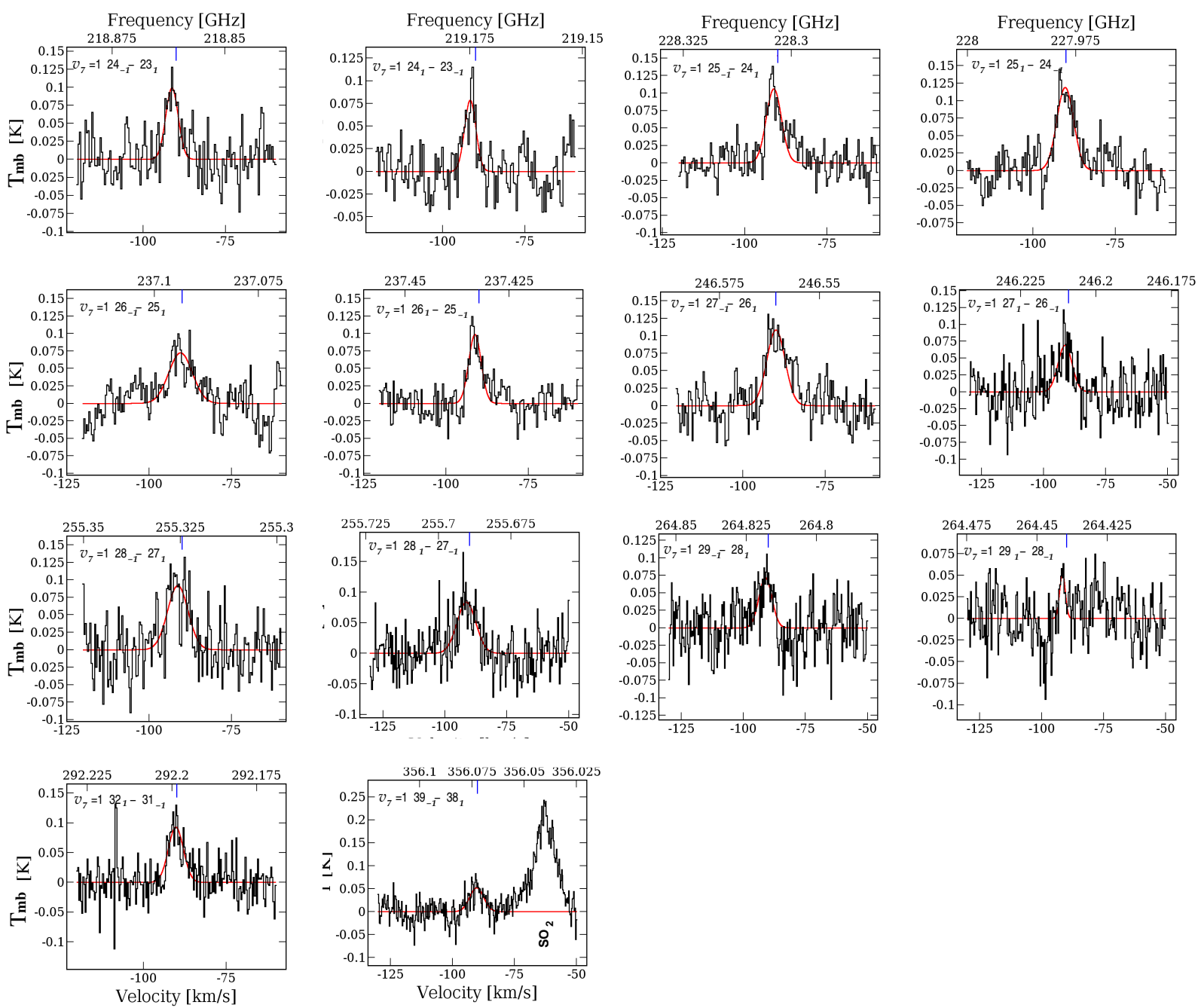

Figure 4. Observed spectra of the detected $\mathrm{HC}_{3} \mathrm{~N} v_{7}=1$ lines. The red curves show the gaussian fits to the lines. The transition are indicated in the top left corner of each panel. The blue line at the top of the spectra indicate the rest frequency of each transition.

ISM but at different transitions, therefore significant contamination is unlikely). b) neighbor lines (nl): criterion that was used to identify the spectral lines which appear within a spectral window equivalent to $V_{\mathrm{lsr}}-90 \pm 30 \mathrm{~km} \mathrm{~s}^{-1}$; c) clean lines (cl): lines whose spectral profiles and FHWH values are not affected by other spectral features, such as blended or neighbor lines.

\subsection{1 $v=0$ lines}

Using the criteria mentioned above, we confirmed the detection of 9 rotational lines of the main $\mathrm{C}$ isotopologue of $\mathrm{HC}_{3} \mathrm{~N}$ in the ground vibrational state $(v=0)$. Six of them were identified in the APEX-1 band, while three lines were identified in the APEX-2 band (see Fig. 2). Identified lines are listed in Table 1 along with some of their spectroscopic parameters like frequency, energy of the upper level of the transition $\left(E_{u}\right)$, and Einstein coefficient of the transition $\left(A_{u l}\right)$. For these lines we performed a Gaussian fit and we obtained their integrated areas $\left(\int T_{\mathrm{mb}} d \mathrm{v}\right)$, linewidths (FWHM), peak temperatures $\left(T_{\text {peak }}\right)$, and velocities $\left(\mathrm{V}_{\mathrm{lsr}}\right)$. These parameters are also indicated in Table 1 . As can be seen from the table, the velocity dispersion of the peak velocities of the lines around the systemic velocity $V_{\mathrm{lsr}}=-90 \mathrm{~km} \mathrm{~s}^{-1}$ is quite low $\left(\leqslant 0.57 \mathrm{~km} \mathrm{~s}^{-1}\right)$, and the mean systemic velocity of the lines is $\overline{\mathrm{V}}_{\mathrm{lsr}}=-90.43 \pm 0.08 \mathrm{~km} \mathrm{~s}^{-1}$. In Table 1 we also indicate the optical depth of each line $(\tau)$ that will be further derived in Section 4.2.1.

It is also worth mentioning that the frequency range analysed in this work covers several transitions of larger cyanopolines (thirty-one for the molecule $\mathrm{HC}_{5} \mathrm{~N}$ and over forty for the molecule $\mathrm{HC}_{7} \mathrm{~N}$ ) and still, no lines corresponding to these species were detected in our dataset.

\subsubsection{Isotopologues}

We also searched for lines of the ${ }^{13} \mathrm{C}-, \mathrm{D}$-, and ${ }^{15} \mathrm{~N}$-bearing isotopologues of $\mathrm{HC}_{3} \mathrm{~N}$ that are predicted to be present in the observed frequency intervals. We identified ${ }^{13} \mathrm{C}$ isotopologue lines with maximum $J$ numbers between 24 and 27, all in the ground vibrational state $v=0$. They are shown in Fig. 3. The transition $(25-24)$ of $\mathrm{H}^{13} \mathrm{CCCN}$ was not detected since it overlaps with the strong ${ }^{13} \mathrm{CO}(2-1)$ line. The same occurs with the $(26-25)$ transition of $\mathrm{HC}^{13} \mathrm{CCN}$, which 
Table 1. $\mathrm{HC}_{3} \mathrm{~N}$ lines detected in G331 and their parameters obtained from Gaussian fits.

\begin{tabular}{|c|c|c|c|c|c|c|c|c|c|}
\hline Transition & $\begin{array}{c}\text { Frequency } \\
(\mathrm{MHz})\end{array}$ & $\begin{array}{l}E_{u} \\
(\mathrm{~K})\end{array}$ & $\begin{array}{c}A_{u l} \\
\left(\times 10^{-5} \mathrm{~s}^{-1}\right)\end{array}$ & $\underset{\left(\mathrm{K} \mathrm{km} \mathrm{s}^{-1}\right)}{\int T_{\mathrm{mb}} d \mathrm{v}}$ & $\begin{array}{c}\mathrm{V}_{\mathrm{lsr}} \\
\left(\mathrm{km} \mathrm{s}^{-1}\right)\end{array}$ & $\begin{array}{c}T_{\text {peak }} \\
(\mathrm{K})\end{array}$ & $\begin{array}{c}\text { FWHM } \\
\left(\mathrm{km} \mathrm{s}^{-1}\right)\end{array}$ & $\tau$ & Remark $^{\S}$ \\
\hline \multicolumn{10}{|l|}{$v=0$} \\
\hline $24-23$ & 218324.72 & 130.98 & 82.6 & $6.94 \pm 0.72$ & $-90.35 \pm 0.05$ & $1.17 \pm 0.05$ & $5.39 \pm 0.11$ & $0.298^{*}$ & $\mathrm{cl}$ \\
\hline $25-24$ & 227418.91 & 141.89 & 93.5 & $7.49 \pm 0.75$ & $-90.37 \pm 0.05$ & $1.23 \pm 0.05$ & $5.57 \pm 0.11$ & $0.278^{*}$ & $\mathrm{cl}$ \\
\hline $26-25$ & 236512.78 & 153.24 & 105.2 & $6.20 \pm 0.62$ & $-90.35 \pm 0.05$ & $0.99 \pm 0.04$ & $5.55 \pm 0.12$ & $0.260^{*}$ & $\mathrm{cl}$ \\
\hline $27-26$ & 245606.31 & 165.03 & 117.9 & $6.02 \pm 0.61$ & $-90.37 \pm 0.07$ & $0.94 \pm 0.06$ & $5.63 \pm 0.17$ & $0.248^{*}$ & $\mathrm{cl}$ \\
\hline $28-27$ & 254699.51 & 177.25 & 131.6 & $6.47 \pm 0.66$ & $-90.37 \pm 0.07$ & $1.02 \pm 0.06$ & $5.58 \pm 0.15$ & $0.235^{*}$ & $\begin{array}{c}\text { pbw }{ }^{33} \mathrm{SO}_{2} \\
\text { nl } \mathrm{CH}_{2} \mathrm{NH}\end{array}$ \\
\hline $29-28$ & 263792.31 & 189.91 & 146.3 & $5.85 \pm 0.59$ & $-90.49 \pm 0.07$ & $0.89 \pm 0.06$ & $5.93 \pm 0.16$ & $0.206^{*}$ & pbw $\mathrm{CH}_{3} \mathrm{OH}$ \\
\hline $37-36$ & 336520.08 & 306.91 & 304.8 & $4.86 \pm 0.50$ & $-90.50 \pm 0.08$ & $0.65 \pm 0.05$ & $6.67 \pm 0.19$ & $0.082^{*}$ & $\mathrm{nl} \mathrm{SO}$ \\
\hline $38-37$ & 345609.01 & 323.49 & 330.2 & $3.87 \pm 0.39$ & $-90.57 \pm 0.06$ & $0.58 \pm 0.04$ & $6.16 \pm 0.14$ & $0.077^{*}$ & pbw $\mathrm{CH}_{3} \mathrm{OH}$ \\
\hline $39-38$ & 354697.46 & 340.51 & 357.1 & $4.14 \pm 0.43$ & $-90.54 \pm 0.08$ & $0.53 \pm 0.04$ & $6.92 \pm 0.18$ & $0.060^{*}$ & $\mathrm{cl}$ \\
\hline \multicolumn{10}{|c|}{${ }^{13} \mathrm{C}$ Isotopologues } \\
\hline \multicolumn{10}{|l|}{$\mathrm{H}^{13} \mathrm{CCCN}$} \\
\hline $26-25$ & 229203.09 & 148.51 & 95.7 & $0.28 \pm 0.04$ & $-91.1 \pm 0.2$ & $0.06 \pm 0.02$ & $4.1 \pm 0.4$ & $0.024^{\dagger}$ & $\mathrm{cl}$ \\
\hline $27-26$ & 238015.68 & 159.93 & 197.3 & $0.30 \pm 0.04$ & $-90.9 \pm 0.2$ & $0.06 \pm 0.02$ & $4.8 \pm 0.5$ & $0.022^{\dagger}$ & $\mathrm{cl}$ \\
\hline \multicolumn{10}{|l|}{$\mathrm{HC}^{13} \mathrm{CCN}$} \\
\hline $24-23$ & 217398.56 & 130.42 & 81.5 & $0.28 \pm 0.05$ & $-89.4 \pm 0.4$ & $0.07 \pm 0.02$ & $4.2 \pm 0.8$ & $0.025^{\dagger}$ & $\mathrm{cl}$ \\
\hline $25-24$ & 226454.18 & 141.29 & 92.3 & $0.32 \pm 0.05$ & $-90.7 \pm 0.4$ & $0.06 \pm 0.02$ & $5.9 \pm 1.0$ & $0.024^{\dagger}$ & $\mathrm{cl}$ \\
\hline $27-26$ & 244564.44 & 164.33 & 116.4 & $0.32 \pm 0.06$ & $-91.5 \pm 0.4$ & $0.07 \pm 0.03$ & $3.6 \pm 0.8$ & $0.027^{\dagger}$ & pbw $\mathrm{CH}_{3} \mathrm{OH}$ \\
\hline \multicolumn{10}{|l|}{$\mathrm{HCC}^{13} \mathrm{CN}$} \\
\hline $24-23$ & 217419.57 & 130.44 & 81.6 & $0.51 \pm 0.06$ & $-90.2 \pm 0.2$ & $0.10 \pm 0.02$ & $5.4 \pm 0.4$ & $0.035^{\dagger}$ & $\begin{array}{c}\text { pbw } \mathrm{CH}_{3} \mathrm{OH} \\
\text { nl }{ }^{13} \mathrm{CN}\end{array}$ \\
\hline $25-24$ & 226476.04 & 141.31 & 92.3 & $0.43 \pm 0.06$ & $-89.9 \pm 0.4$ & $0.08 \pm 0.03$ & $5.4 \pm 0.7$ & $0.031^{\dagger}$ & $\mathrm{cl}$ \\
\hline $26-25$ & 235532.20 & 152.61 & 103.9 & $0.50 \pm 0.07$ & $-88.9 \pm 0.3$ & $0.09 \pm 0.02$ & $5.6 \pm 0.6$ & $0.037^{\dagger}$ & $\mathrm{cl}$ \\
\hline \multicolumn{10}{|l|}{$v_{7}=1$} \\
\hline $24_{-1}-23_{1}$ & 218860.81 & 452.15 & 82.7 & $0.58 \pm 0.07$ & $-91.3 \pm 0.3$ & $0.10 \pm 0.03$ & $4.9 \pm 0.5$ & $0.015^{*}$ & $\mathrm{cl}$ \\
\hline $24_{1}-23_{-1}$ & 219173.75 & 452.34 & 83.0 & $0.34 \pm 0.05$ & $-91.6 \pm 0.3$ & $0.08 \pm 0.02$ & $4.1 \pm 0.6$ & $0.018^{*}$ & $\mathrm{cl}$ \\
\hline $25_{1}-24_{-1}$ & 227977.27 & 463.09 & 93.5 & $0.69 \pm 0.08$ & $-90.2 \pm 0.2$ & $0.12 \pm 0.02$ & $6.0 \pm 0.5$ & $0.013^{*}$ & $\mathrm{cl}$ \\
\hline $25_{-1}-24_{1}$ & 228303.17 & 463.29 & 93.4 & $0.70 \pm 0.09$ & $-90.8 \pm 0.3$ & $0.10 \pm 0.02$ & $6.8 \pm 0.6$ & $0.011^{*}$ & $\mathrm{cl}$ \\
\hline $26_{-1}-25_{1}$ & 237093.38 & 474.47 & 105.2 & $0.53 \pm 0.07$ & $-90.5 \pm 0.4$ & $0.07 \pm 0.02$ & $8.4 \pm 1.2$ & $0.009^{*}$ & $\mathrm{cl}$ \\
\hline $26_{1}-25_{-1}$ & 237432.26 & 474.69 & 105.7 & $0.57 \pm 0.07$ & $-91.0 \pm 0.2$ & $0.10 \pm 0.03$ & $5.3 \pm 0.5$ & $0.014^{*}$ & $\mathrm{cl}$ \\
\hline $27_{1}-26_{-1}$ & 246209.14 & 486.29 & 118.0 & $0.45 \pm 0.08$ & $-91.4 \pm 0.5$ & $0.07 \pm 0.03$ & $9.0 \pm 1.9$ & $0.011^{*}$ & $\mathrm{cl}$ \\
\hline $27-1-26_{1}$ & 246560.95 & 486.52 & 118.5 & $0.78 \pm 0.10$ & $-89.3 \pm 0.4$ & $0.10 \pm 0.03$ & $8.1 \pm 0.9$ & $0.009^{*}$ & $\mathrm{cl}$ \\
\hline $28_{-1}-27_{1}$ & 255324.55 & 498.54 & 131.7 & $0.58 \pm 0.10$ & $-91.5 \pm 0.5$ & $0.09 \pm 0.03$ & $6.7 \pm 1.1$ & $0.011^{*}$ & $\mathrm{cl}$ \\
\hline $28_{1}-27-1$ & 255689.29 & 498.79 & 132.2 & $0.72 \pm 0.11$ & $-91.3 \pm 0.6$ & $0.08 \pm 0.02$ & $8.9 \pm 1.6$ & $0.008^{*}$ & $\mathrm{cl}$ \\
\hline $29_{1}-28-1$ & 264439.58 & 511.23 & 146.4 & $0.20 \pm 0.04$ & $-91.6 \pm 0.3$ & $0.05 \pm 0.03$ & $2.6 \pm 0.7$ & $0.029^{*}$ & $\mathrm{cl}$ \\
\hline $29_{-1}-28_{1}$ & 264817.24 & 511.50 & 147.1 & $0.40 \pm 0.07$ & $-91.1 \pm 0.5$ & $0.07 \pm 0.02$ & $6.2 \pm 1.1$ & $0.012^{*}$ & $\mathrm{cl}$ \\
\hline $32_{1}-31_{-1}$ & 292198.64 & 552.26 & 197.9 & $0.50 \pm 0.08$ & $-90.2 \pm 0.3$ & $0.09 \pm 0.02$ & $4.9 \pm 0.6$ & $0.014^{*}$ & $\mathrm{cl}$ \\
\hline $39-1-38_{1}$ & 356072.44 & 662.68 & 359.2 & $0.31 \pm 0.06$ & $-90.3 \pm 0.4$ & $0.05 \pm 0.02$ & $6.6 \pm 1.0$ & $0.008^{*}$ & nlo $\mathrm{SO}_{2}$ \\
\hline
\end{tabular}

* Obtained iteratively from the opacity correction (Sect 4.2.1) † Obtained from Eq.8 (Sect. 4.2.3)

seems to overlap with another strong $\left(T_{\mathrm{mb}} \sim 1.3 \mathrm{~K}\right)$ unidentified line. Thus, a complete triad $\mathrm{H}^{13} \mathrm{CCCN}-\mathrm{HC}^{13} \mathrm{CCN}-$ $\mathrm{HCC}^{13} \mathrm{CN}$ could not be detected for a particular $J$ transition. As a marginal detection, we included the $\mathrm{HC}^{13} \mathrm{CCN}$ (27-26) line, which is better identified over an extra hanningsmoothed spectra (not shown here).

The velocity dispersion of the derived peak velocity of the lines with respect to the systemic velocity $\left(\leqslant 1.5 \mathrm{~km} \mathrm{~s}^{-1}\right)$ is larger than that of the main isotopologue, probably due to a lower accuracy of the fit. The mean systemic velocity of the lines is $\overline{\mathrm{V}}_{\mathrm{lsr}}=-90.3 \pm 0.8 \mathrm{~km} \mathrm{~s}^{-1}$. No transitions for the $\mathrm{DC}_{3} \mathrm{~N}$ and $\mathrm{HC}_{3}^{15} \mathrm{~N}$ isotopologues were detected in our dataset.

\section{$4.1 .3 v_{7}=1$ lines}

The molecule of $\mathrm{HC}_{3} \mathrm{~N}$ has seven modes of vibration, three bending modes (doubly degenerate) and four stretching modes, which are usually identified from $v_{1}$ to $v_{7}$ (for a complete revision, see Wyrowski et al. 1999). Then, besides rotational lines in the ground vibrational state $(v=0)$, we also searched for rotational transitions of $\mathrm{HC}_{3} \mathrm{~N}$ in vibrationally excited states. As a result, we found transitions in the lowest 
excited state, $v_{7}=1$ (corresponding to the bending vibration mode of the $\mathrm{C}-\mathrm{C} \equiv \mathrm{C}$ group). The detected lines are shown in Fig. 4. The interaction between the bending angular momentum of the vibrational $v_{7}=1$ state and the rotational angular momentum of the molecule leads to a splitting in two rotational levels for each $J \rightarrow J-1$ transition (indicated in Fig. 4 as -1 and 1). As for the case of the $\mathrm{HC}^{13} \mathrm{CCN}$ (27-26) line (see Sect. 4.1.2), we included the marginal detection of the transition $29_{1}-28_{-1}$. It should be noted that the transition $39_{1}-38_{-1}$ was not detected since it is blended with an intense $(\sim 0.25 \mathrm{~K})$ and wide $\left(\sim 8 \mathrm{~km} \mathrm{~s}^{-1}\right)$ unidentified line at $\sim 355.569 \mathrm{GHz}$. The spectroscopic and Gaussian fit parameters are listed in Table 1 . In particular, most of them have the same transitional levels of $\mathrm{HC}_{3} \mathrm{~N} v=0$ with maximum $J$ numbers between 24 and 39. A velocity dispersion of $\leq 1.6 \mathrm{~km} \mathrm{~s}^{-1}$ for the derived peak velocities of the lines is observed around the systemic velocity of the source, which has a mean value of $\overline{\mathrm{V}}_{\mathrm{lsr}}=-90.9 \pm 0.7 \mathrm{~km} \mathrm{~s}^{-1}$. For consistency, we performed the Gaussian fit to the lines using the velocity interval defined for the $25_{1}-24_{-1}$ transition, which is the most conspicuous one.

\subsection{Line analysis}

\subsubsection{LTE analysis}

In order to estimate excitation temperatures, $T_{\text {exc }}$, and column densities, $N\left(\mathrm{HC}_{3} \mathrm{~N}\right)$, we constructed population diagrams from the identified transitions of the molecule. This term refers to a plot of the column density per statistical weight of a number of transition energy levels, as a function of their energy above the ground state (Goldsmith \& Langer 1999). In local thermodynamical equilibrium (LTE), and assuming that the emission is optically thin, this corresponds to the Boltzmann distribution and can be represented as

$$
\ln \left(\frac{N_{u}}{g_{u}}\right)=\ln \left(\frac{N_{\mathrm{tot}}}{Q\left(T_{\mathrm{exc}}\right)}\right)-\frac{E_{u}}{k T_{\mathrm{exc}}}
$$

where $N_{u} / g_{u}$ is the column density per statistical weight for the upper level $u, E_{u}$ is the energy of the upper level, $Q\left(T_{\text {exc }}\right)$ is the partition function, and $N_{\text {tot }}$ is the total column density. Since $N_{u}$ can be estimated from the integrated intensity of the line as

$$
N_{u}=\frac{8 \pi k \nu^{2}}{h c^{3} A_{u l}} \times \int T_{\mathrm{mb}} d \mathrm{v}
$$

then, Eq. 1 represents a Boltzmann distribution whose values $\ln \left(N_{u} / g_{u}\right)$ vs. $E_{u}$ can be obtained from the lines and can be fitted using a straight line, whose slope is defined by the term $1 / T_{\text {exc }}$.

The population diagrams were constructed using a beam dilution factor $\ln \left(\frac{\Delta \Omega_{s}}{\Delta \Omega_{a}+\Delta \Omega_{s}}\right)$ where $\Delta \Omega_{a}$ and $\Delta \Omega_{s}$ are the solid angle of the antenna beam and the solid angle of the source, respectively. The beam dilution factor was applied on the left side of Eq. 1. A source size of $\sim 6$ arcsec was adopted (see Sect. 4.2.2).

In the upper panel of Fig. 5 we show the obtained population diagrams for the $\mathrm{HC}_{3} \mathrm{~N} v=0$ lines. From the excellent fit obtained from the data $\left(\chi_{\text {red }}^{2}=1.28\right)$ we derived $N\left(\mathrm{HC}_{3} \mathrm{~N}\right)=(5.8 \pm 0.6) \times 10^{14} \mathrm{~cm}^{-2}$ and $T_{\text {exc }}=89 \pm 4 \mathrm{~K}$. The
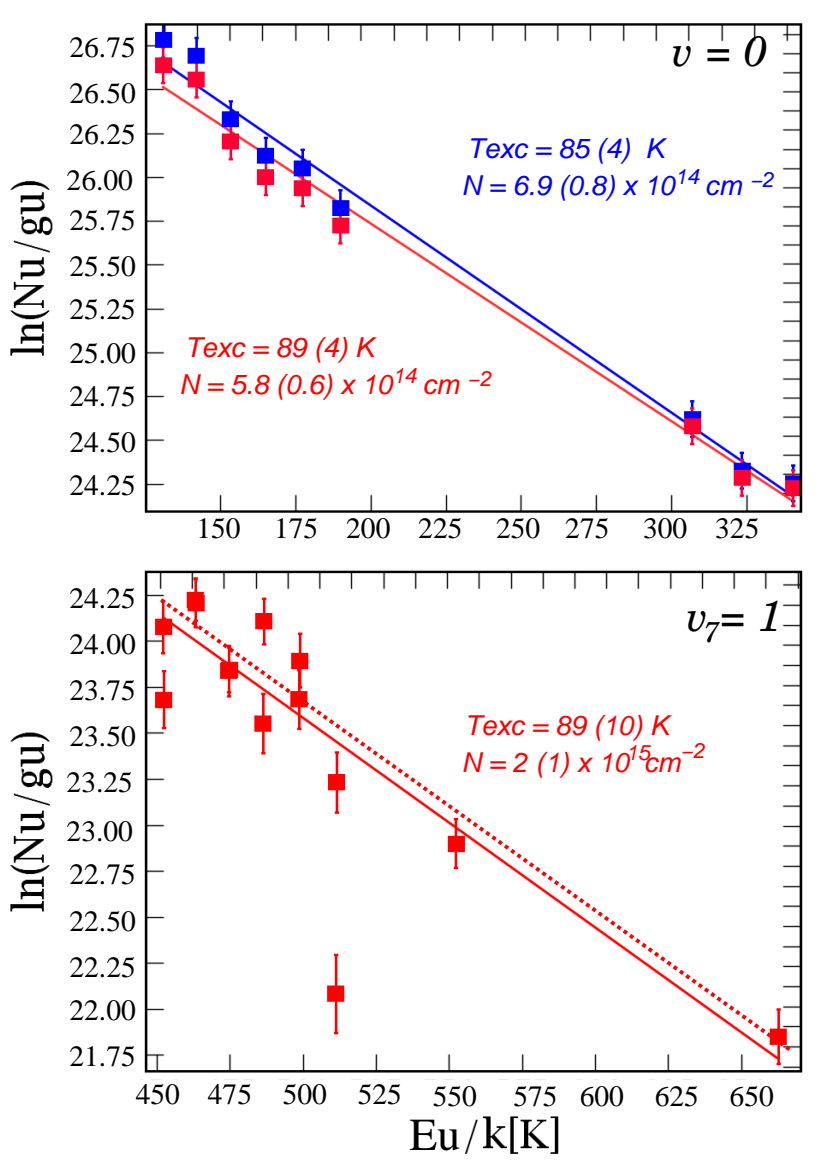

Figure 5. Upper panel: Population diagrams for the $\mathrm{HC}_{3} \mathrm{~N} v=0$ lines, showing the least squares fits without optical depth correction (red color) and with optical depth correction (blue color). The corresponding errors in density and temperature are indicated in parenthesis. Lower panel: Population diagrams for the $\mathrm{HC}_{3} \mathrm{~N} v_{7}=1$ lines. The upper dotted line represents the least square fit without the transition line $29_{1-28}-1$ (see transitions with $E_{u}=511 \mathrm{~K}$ ).

plotted uncertainties in Fig. 5 are:

$$
\Delta\left(\ln \frac{N_{u}}{g_{u}}\right)=\frac{\Delta W}{W}
$$

were $W$ is the integrated area of the line and $\Delta W$ is its uncertainty, estimated as ${ }^{9}$

$$
\Delta W=\sqrt{(\mathrm{cal} / 100 \times W)^{2}+(r m s \sqrt{2 \times \mathrm{FWHM} \times \Delta \mathrm{v}})^{2}}
$$

being cal the calibration error (in percentage), rms the noise around the selected line (in K), FWHM the line full width half maximum (in $\mathrm{km} \mathrm{s}^{-1}$ ), and $\Delta \mathrm{v}$ the bin size (in $\mathrm{km} \mathrm{s}^{-1}$ )

The population diagrams obtained before were constructed under assumption of optically thin emission $(\tau<<1)$. In order to test the reliability of this approximation, we have introduced the optical depth correction factor

9 http://cassis.irap.omp.eu/docs/RadiativeTransfer.pdf 
$C_{\tau}$ (photon escape probability) in Eq. 2 as

$$
N_{u}=\frac{8 \pi k \nu^{2}}{h c^{3} A_{u l}} \times C_{\tau} \times \int T_{\mathrm{mb}} d \mathrm{v}
$$

where $C_{\tau}=\tau /\left(1-e^{-\tau}\right)$ (Goldsmith \& Langer 1999), being $\tau$ the optical depth of the line. When the line is optically thin, the factor $C_{\tau}$ is then equal to unity. Thus, Eq. 1 becomes

$$
\ln \left(\frac{N_{u}}{g_{u}}\right)=\ln \left(\frac{N_{\text {tot }}}{Q\left(T_{\text {exc }}\right)}\right)-\ln C_{\tau}-\frac{E_{u}}{k T_{\text {exc }}},
$$

and the ordinate of the population diagram fit is rescaled to slightly larger values. The correction is performed with CASSIS, which execute iteratively the calculations of $C_{\tau}$ until values of temperature and column density converge. From the fit $\left(\chi_{\text {red }}^{2}=1.33\right)$ we derived a column density $N\left(\mathrm{HC}_{3} \mathrm{~N}\right)=(6.9 \pm 0.8) \times 10^{14} \mathrm{~cm}^{-2}$ and a excitation temperature $T_{\text {exc }}=85 \pm 4 \mathrm{~K}$. Then, the percentage differences of $N\left(\mathrm{HC}_{3} \mathrm{~N}\right)$ and $T_{\text {exc }}$ with respect to the values obtained without optical depth correction are about 19 percent higher and 5 percent lower, respectively. For the sake of comparison, the corrected population diagram is shown in Fig. 5 together with the original diagram without optical depth correction. The optical depth of each line, estimated with this method, is listed in Table 1. As can be noticed from Table 1 and Fig. 5, differences are moderately significant $(\tau \gtrsim 0.2)$ only for low $J$-values $(J<28)$. This trend is in line with the variation of the optical depth as a function of $J$ presented by Goldsmith \& Langer (1999) for the molecule of $\mathrm{HC}_{3} \mathrm{~N}$ with $h \mathrm{~B} / k T=0.003$, for which a maximum relative optical depth is obtained for $J \approx 20$, and monotonically decreasing for $J \lesssim 20$ and $J \gtrsim 20$ (see fig. 1 of that work).

As can be noticed from Fig. 2 some lines show Lorentzian instead of Gaussian profiles, which is a very usual feature in emission lines affected by outflows. After a Lorentzian fit of the beam-diluted lines, we derived an opacity-corrected column density $N\left(\mathrm{HC}_{3} \mathrm{~N}\right)=(7.8 \pm 0.5) \times 10^{14} \mathrm{~cm}^{-2}$ and an excitation temperature $T_{\text {exc }}=90 \pm 3 \mathrm{~K}$, respectively, which means a difference of $\sim 13$ percent higher in density and $\sim 7$ percent also higher in temperature, compared with values obtained by Gaussian fitting.

In the lower panel of Fig. 5 we also show the population diagrams obtained for the $v_{7}=1$ lines. From the fit we derived a column density and temperature of $N\left(\mathrm{HC}_{3} \mathrm{~N}\right)=(2.2 \pm 2.0) \times 10^{15} \mathrm{~cm}^{-2}$ and $T_{\text {exc }}=87 \pm 14 \mathrm{~K}$, respectively. The low accuracy of the fit $\left(\chi_{\text {red }}^{2}=6.07\right)$ is due, not only to the significantly lower signal-to-noise ratio of the detections, but mostly to the transition line $29_{1}-28_{-1}$ $\left(E_{u}=511.23 \mathrm{~K}\right)$ which lies outside the $3 \mathrm{rms}$ scatter limit of the plot. Removing this line from the plot, we obtained a more accurate fit $\left(\chi_{\text {red }}^{2}=2.8\right)$; the column density and temperature obtained then are $N\left(\mathrm{HC}_{3} \mathrm{~N}\right)=(2 \pm 1) \times 10^{15} \mathrm{~cm}^{-2}$ and $T_{\text {exc }}=89 \pm 10 \mathrm{~K}$, respectively. As expected, these values are consistent with those obtained for the $v=0$ lines. Optical depths obtained iteratively for the $v_{7}=1$ lines are $<0.03$ (see Table 1), which amounts to a difference of only 0.6 percent and 3.5 percent in temperature and column density, respectively, with respect to those obtained without opacity correction (not shown in Fig. 5). The excitation temperatures derived before for the rotational transitions in the $v=0$ and $v_{7}=1$ states are in good agreement with those derived from the $\mathrm{CH}_{3} \mathrm{OH}$ emission by Mendoza et al. (2018b) and from
$\mathrm{CH}_{3} \mathrm{CCH}$ emission by Hervías-Caimapo et al. (2019) for the ambient gas in G331. We note, however, that $\mathrm{HC}_{3} \mathrm{~N}$ column densities derived above for G331 are almost one order of magnitude larger than some found in typical Galactic hot molecular cores (e.g. Taniguchi et al. 2018a,c).

Besides transitions of different $J$ levels in the same vibrational state, the level population of different $v$ states for a constant rotational $J$ level can also be analyzed to derive the so-called "vibrational temperature" $\left(T_{\text {vib }}\right)$ which determines the relative population of vibrationally excited levels. A rough estimate of $T_{\text {vib }}$ can be obtained from a comparision between vibrationally excited lines with ground-state lines (e.g. Goldsmith et al. 1982). Here, assuming optically thin emission, we use

$$
\frac{W_{\left(v_{7}=1\right)}}{W_{(v=0)}}=\exp \left(\frac{-\Delta E_{70}}{k T_{\mathrm{vib}}}\right)
$$

where $W_{\left(v_{7}=1\right)}$ and $W_{(v=0)}$ stand for the integrated intensities of a $J \rightarrow J-1$ transition for the vibrational states $v_{7}=1$ and $v=0$, respectively, and $\Delta E_{70}$ is the energy difference between the $v=0$ and $v_{7}=1$ states. Thus, we estimated $T_{\mathrm{vib}}$ for constant $J$ levels in the range between 24 to 27 and we obtained vibrational temperatures in the range $130 \mathrm{~K}$ to $145 \mathrm{~K}$. As expected, obtained values of $T_{\mathrm{vib}}$ are higher than those previously obtained, for rotational transitions within the same vibrational state, from the population diagrams (a similar trend was also reported by Goldsmith et al. 1982 in the Orion Molecular Cloud using $v_{7}=1$ and $v=0$ lines of $\mathrm{HC}_{3} \mathrm{~N}$ ) but lower than those reported in the literature for Galactic hot cores and star forming regions (e.g. Wyrowski et al. 1999; Jiménez-Serra et al. 2009; Peng et al. 2017). It is worth mentioning that the above values of $T_{\text {vib }}$ should be considered as lower limits since the $v=0$ state is expected to be dominated by more extended and cooler emission. Thus, the ratio $W_{\left(v_{7}=1\right)} / W_{(v=0)}$ is rather a measure of the relative population in the $v_{7}=1$ vibrational state instead of a measure of $T_{\mathrm{vib}}$ (Wyrowski et al. 1999). Observations of more vibrational modes, either bending or stretching, are needed in order to determine $T_{\text {vib }}$ more accurately.

\subsubsection{Non-LTE analysis using collision rates}

All the estimates obtained so far rely on the assumption of LTE regime. By means of the excitation diagrams displayed in Fig. 5, we obtained single excitation temperatures from the whole set of observed rotational lines of $\mathrm{HC}_{3} \mathrm{~N}$, both in ground and vibrationally excited states. The Boltzmann distribution of the excitation diagrams and the quality of the straight lines (with slope $1 / T_{\text {exc }}$, Eq. 6) seem to validate the LTE condition for rotational transitions of $\mathrm{HC}_{3} \mathrm{~N}$. As a consequence, the kinetic temperatures would be expected to be similar to the excitation temperatures if actually all the levels were thermalized.

For a better understanding of the excitation mechanisms and optical depth effects, as to determine the gas kinetic temperature and density, it would also be useful to carry out an analysis of the $\mathrm{HC}_{3} \mathrm{~N}$ emission assuming non-LTE conditions. To this end, we use statistical equilibrium calculations to perform non-LTE models of the $\mathrm{HC}_{3} \mathrm{~N}$ $(\nu=0)$ lines. The statistical equilibrium calculations were 
carried out using the RADEX $\operatorname{code}^{10}$ (van der Tak et al. 2007). Then, by means of non-local radiation effects we tested how far is the $\mathrm{HC}_{3} \mathrm{~N}$ emission from the LTE hypothesis considering collisional excitations induced by molecular hydrogen, whose rate coefficients are presented and discussed in Faure et al. (2016). The RADEX calculations were computed simultaneously applying the Markov Chain Monte Carlo method (MCMC) (Foreman-Mackey et al. 2013), ${ }^{11}$ which is hereafter referred to as RADEX/MCMC. In general, the RADEX/MCMC method computes the best solution by means of numerical chains in a n-dimensional parameter space that can be delimited by different free parameters, such as the kinetic temperature $\left(T_{\text {kin }}\right)$, column density $(N)$, linewidths (FWHM), source size, and volume density $\left(n\left(\mathrm{H}_{2}\right)\right)$. Taking into account the availability of collision rate coefficients, the last parameter is set accordingly to the $\mathrm{H}_{2}-\mathrm{HC}_{3} \mathrm{~N}$ collision system (e.g. Faure et al. 2016). Through stochastic processes, the RADEX/MCMC finds the best solution up to achieve the minimum value of the $\chi^{2}$ function. Since the method operates using the spectral lines as main input, the quality of the models depends on the spectral survey (e.g. number and strength of the lines), the free parameters and their ranges of integration.

The RADEX/MCMC models of $\mathrm{HC}_{3} \mathrm{~N}$ were computed considering the first six rotational lines listed in Table 1, from $J=24-23$ to $J=29-28$. As free parameters, we introduced and calculated the distributions of $T_{\text {kin }}, N\left(\mathrm{HC}_{3} \mathrm{~N}\right)$, $n\left(\mathrm{H}_{2}\right)$, and source size. In order to encompass the $\mathrm{HC}_{3} \mathrm{~N}$ emission, the statistical equilibrium calculations were carried out assuming an emitting region $\leq 6$ arcsec in size, a value consistent with the $\mathrm{HC}_{3} \mathrm{~N} J=38-37$ emission map exhibited in Hervías-Caimapo et al. (2019). Since the quality of the computation is a monotonically increasing function of the numbers of steps or iterations, we included in our routines large samples for draw numbers, of the order of $10^{4}$.

As a corner diagram, the RADEX/MCMC results of $\mathrm{HC}_{3} \mathrm{~N}$ are shown in Fig. 6. In that plot, the histograms of each free parameter merge into bi-dimensional diagrams exhibiting the highest probability distribution. Distributions of the $\mathrm{HC}_{3} \mathrm{~N}$ column density, $\mathrm{H}_{2}$ density, kinetic temperature and source size are exhibited. Summarizing, the physical conditions derived from the best RADEX/MCMC computation $\left(\chi_{r e d}^{2}=4.41\right)$, were obtained to be $N\left(\mathrm{HC}_{3} \mathrm{~N}\right)=4.5 \pm 0.1 \times 10^{14} \mathrm{~cm}^{-2}$ and $T_{\text {kin }}=90 \pm 3 \mathrm{~K}$ for a central core with size and $\mathrm{H}_{2}$ volume density of $\lesssim 6$ arcsec and $\sim 2 \times 10^{7} \mathrm{~cm}^{-3}$, respectively.

In comparison with the LTE analysis of $\mathrm{HC}_{3} \mathrm{~N}$, the results obtained assuming non-LTE conditions are relatively in good agreement with those obtained by the excitation diagram for the $v=0$ state (see Sect. 4.2.1). The percentage differences of column density and temperature with respect to the values obtained in LTE conditions for the $v=0$ lines with optical depth correction are about 6 percent higher and 53 percent lower, respectively, while the difference with respect to the values without optical depth correction is only about 1 percent higher and 28 percent lower, respectively.

\footnotetext{
10 https://home.strw.leidenuniv.nl/ moldata/radex.html
}

11 http://dfm.io/emcee/current/
Table 2. Physical properties derived for the $\mathrm{HC}_{3} \mathrm{~N}$ and its ${ }^{13} \mathrm{C}$ isotopologues.

\begin{tabular}{lccc}
\hline Isotopologue & $\begin{array}{c}N \\
{\left[\times 10^{13} \mathrm{~cm}^{-2}\right]}\end{array}$ & $\begin{array}{c}X \\
{\left[\times 10^{-11}\right]}\end{array}$ & ${ }^{12} \mathrm{C} /{ }^{13} \mathrm{C}$ \\
\hline $\mathrm{HC}_{3} \mathrm{~N}$ & $69 \pm 8^{\dagger}$ & $79-62$ & - \\
$\mathrm{HCC}^{13} \mathrm{CN}$ & $6.7 \pm 0.9$ & $7.8-5.9$ & $\sim 10$ \\
$\mathrm{HC}^{13} \mathrm{CCN}$ & $3.8 \pm 0.8$ & $4.7-3.1$ & $\sim 18$ \\
$\mathrm{H}^{13} \mathrm{CCCN}$ & $3.7 \pm 0.6$ & $4.4-3.2$ & $\sim 19$ \\
${ }^{13} \mathrm{C} \mathrm{HC}$ N & $3.3 \pm 0.2^{\ddagger}$ & $3.6-3.2$ & $\sim 21$ \\
\hline
\end{tabular}

†Obtained by population diagram (see Sect. 4.2.1)

$\ddagger$ Obtained by the LTE/MCMC method

\subsubsection{Isotopic analysis}

Since a scarce number of transitions from isotopic species were detected, and some of them having a poor signal-tonoise ratio, a population diagram does not provide reliable solutions for the temperature and column density. Then, we estimated the column density of the three detected ${ }^{13} \mathrm{C}$ isotopologues from a single line of each species assuming LTE conditions and using the following formulae:

$$
\tau=-\ln \left[1-\frac{T_{\mathrm{mb}}}{f\left[J\left(T_{\mathrm{exc}}\right)-J\left(T_{\mathrm{bg}}\right)\right]}\right]
$$

being

$$
J(T)=\frac{h \nu}{k}\left[e^{\left(\frac{h \nu}{k T}\right)}-1\right]^{-1},
$$

and

$N=\tau \frac{3 h \Delta v}{8 \pi^{3}} \sqrt{\frac{\pi}{4 \ln 2}} \frac{Q}{\mu^{2}\left(J_{l}+1\right)} e^{\left(\frac{E_{l}}{k T_{\mathrm{exc}}}\right)}\left[1-e^{\left(-\frac{h \nu}{k T_{\mathrm{exc}}}\right)}\right]^{-1}$

(Goldsmith \& Langer 1999), where $T_{\mathrm{mb}}$ stands for the main beam temperature of the line, $f$ for the beam filling factor (estimated to be $\sim 0.035^{12}$ ), $T_{\text {exc }}$ is the excitation temperature (assumed to be $85 \mathrm{~K}$; see Sect. 4.2.1), and $T_{\mathrm{bg}}$ is the background temperature $(2.7 \mathrm{~K})$. For this equation, we estimate an error of about 15-25 percent arising mostly from the uncertainties in $T_{\mathrm{mb}}$ (see Table 1). In Eq. $10 \Delta v$ is the line width FWHM (in $\mathrm{cm} \mathrm{s}^{-1}$ ), $Q$ is the partition function estimated as $Q=k T_{\text {exc }} / h \mathrm{~B}$ (B is the rotational constant of $\left.\mathrm{HC}_{3} \mathrm{~N} ; 4549.059 \mathrm{MHz}\right), \mu$ is the permanent electric dipole of $\mathrm{HC}_{3} \mathrm{~N}$ (assumed to be $3.73172 \times 10^{-18} \mathrm{esu} \mathrm{cm}$; DeLeon \& Muenter 1985), $E_{l}$ (in erg) is the energy of the lower rotational level, and $J_{l}$ is the lower rotational quantum number. Obtained column density for each isotopologue is given in Table 2, while optical depths obtained with Eq. 8 are listed in Table 1. The errors estimated for the column density of the ${ }^{13} \mathrm{C}$ isotopologues were derived considering the uncertainties in $\tau$ (see above) and $\Delta v$ (see Table 1 ). For the calculations, we used the most conspicuous line detected for each isotopic species. Then, for $\mathrm{HCC}^{13} \mathrm{CN}$ and $\mathrm{HC}^{13} \mathrm{CCN}$ we used the (24-23) transition, while for $\mathrm{H}^{13} \mathrm{CCCN}$ we used the (26-25) transition.

12 Estimated considering a source size of $\sim 6$ arcsec (see Sect. 4.2.2) and an average beam size of $\sim 27$ arcsec for the APEX-1 band 


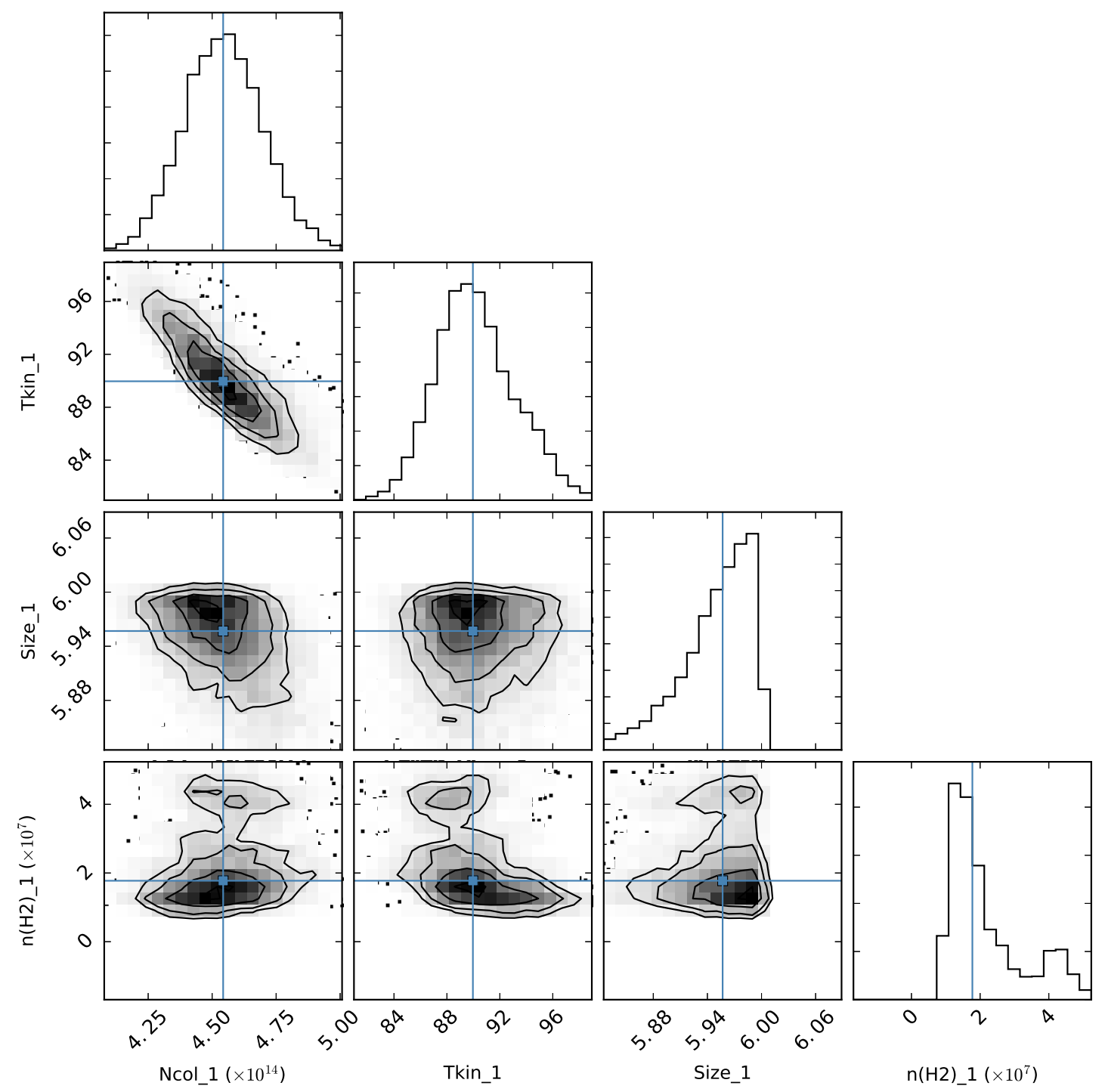

Figure 6. Corner diagram with the highest probability distribution of the column density $\left(\mathrm{Ncol}, \mathrm{cm}^{-2}\right)$, kinetic temperature (Tkin, $\mathrm{K}$ ), source size (Size, arcsec) and $\mathrm{H}_{2}$ density $\left(\mathrm{n}(\mathrm{H} 2), \mathrm{cm}^{-3}\right)$ derived from the best RADEX/MCMC model $\left(\chi_{\text {red }}^{2}=4.41\right)$ of $\mathrm{HC}_{3} \mathrm{~N}$. Collision excitation coefficients between $\mathrm{HC}_{3} \mathrm{~N}$ and $\mathrm{H}_{2}$ were used to perform the calculations considering the $\mathrm{HC}_{3} \mathrm{~N}$ lines from $J=24-23$ to $J=29-28$. The blue solid lines indicate the median values.

The relative abundances of the isotopic species with respect to $\mathrm{H}_{2}$ were derived using the $\mathrm{H}_{2}$ column density obtained previously from the emission of $\mathrm{H}^{13} \mathrm{CO}^{+}$(e.g. Merello et al. 2013a; Mendoza et al. 2018b). ALMA observations of the $\mathrm{H}^{13} \mathrm{CO}^{+}(4-3)$ emission have shown the existence of an internal cavity surrounded by a shocked shell, around the driving source, confined in $\lesssim 5$ arcsec (Merello et al. 2013a) for which an averaged column density $N\left(\mathrm{H}^{13} \mathrm{CO}^{+}\right) \approx 3.2 \pm 0.2 \times 10^{13} \mathrm{~cm}^{-2}$ was derived (result obtained by Hervías-Caimapo et al. 2019, but not included in that work). Then, we estimated the $\mathrm{H}_{2}$ column density using the abundance ratio $\mathrm{H}^{13} \mathrm{CO}^{+} / \mathrm{H}_{2}=3.3 \times 10^{-11}$ of Orion $\mathrm{KL}$ (Blake et al. 1987) and we obtained $N\left(\mathrm{H}_{2}\right)=9.7 \times 10^{23} \mathrm{~cm}^{-2}$, almost one order of magnitude higher than that derived in typical high-mass star forming regions (e.g. Taniguchi et al. 2018b). The abundances of $\mathrm{HC}_{3} \mathrm{~N}, \mathrm{HCC}^{13} \mathrm{CN}, \mathrm{HC}^{13} \mathrm{CCN}$, and $\mathrm{H}^{13} \mathrm{CCN}$ were then obtained dividing their column densities by $N\left(\mathrm{H}_{2}\right)$. In Table 2 we summarize the range of obtained abundances, indicated as $X$, which were derived considering the uncertainties of column densities estimated using Eq. 10. It is worth pointing out that $N\left(\mathrm{H}_{2}\right)$ can be otherwise estimated from the surface density derived by Merello et al. (2013a) from the $1.2 \mathrm{~mm}$ continuum emission of G331 (referred to as MM3 in that work). Adopting a mean molecular weight per Hydrogen molecule $\mu=2.29$ (corrected for Helium), a column density $N\left(\mathrm{H}_{2}\right)=2.7 \times 10^{23} \mathrm{~cm}^{-2}$ can be obtained, which would increase the abundances reported in Table 2 by a factor of $\sim 3.5$

Since $\mathrm{HC}_{3} \mathrm{~N}$ and its ${ }^{13} \mathrm{C}$ isotopologue lines are optically thin (see Col. 9 of Table 1) we can obtain reliable mea- 


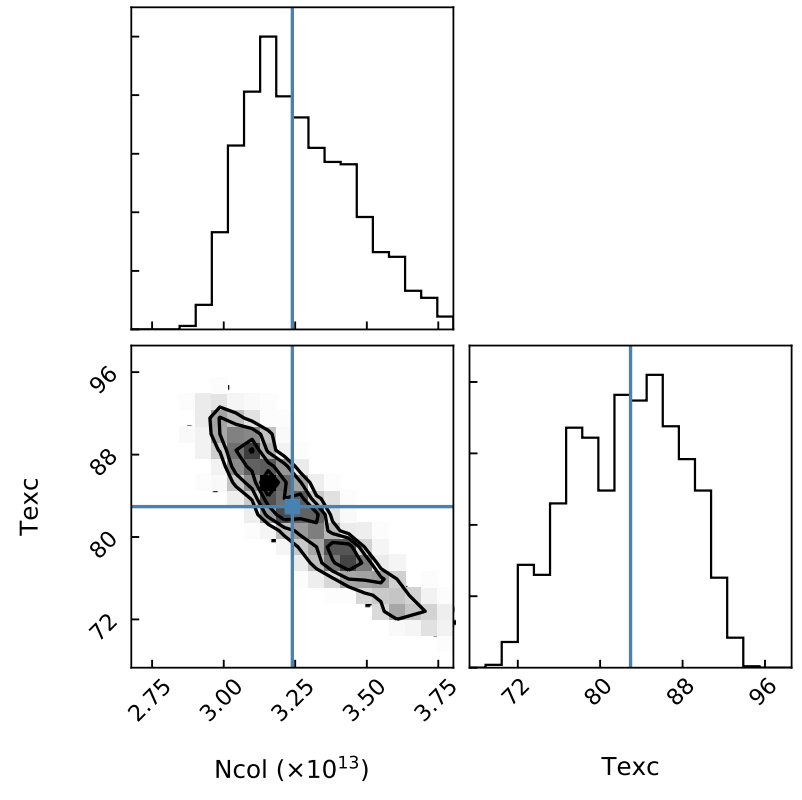

Figure 7. Corner diagram with the highest probability distributions of the column density $\mathrm{N}_{\mathrm{col}}\left(\mathrm{cm}^{-2}\right)$ against the excitation temperature $\mathrm{T}_{\text {exc }}(\mathrm{K})$ derived from the LTE/MCMC models of the ${ }^{13} \mathrm{C} \quad \mathrm{HC}_{3} \mathrm{~N}$ isotopologues. The best model $\left(\chi_{\text {red }}^{2}=5.88\right)$ yielded $T_{\text {exc }}=82 \pm 5 \quad \mathrm{~K}$ and $N\left(\mathrm{H}^{13} \mathrm{CCCN}\right)=N\left(\mathrm{HC}^{13} \mathrm{CCN}\right)=N\left(\mathrm{HCC}^{13} \mathrm{CN}\right)=(3.3 \pm 0.2) \times 10^{13} \mathrm{~cm}^{-2}$ for a source size of 6 arcsec. The blue solid lines indicate the median values.

surements of the ${ }^{12} \mathrm{C} /{ }^{13} \mathrm{C}$ isotopic ratio in $\mathrm{G} 331$ from the column densities calculated before. For the estimations we have considered column densities of $\mathrm{HC}_{3} \mathrm{~N}$ and its isotopologues obtained in LTE conditions. The obtained ${ }^{12} \mathrm{C} /{ }^{13} \mathrm{C}$ isotopic ratios are shown in the last column of Table 2 .

In addition to the estimates obtained above, we also carried out LTE models of the ${ }^{13} \mathrm{C}$ isotopologues of $\mathrm{HC}_{3} \mathrm{~N}$ applying the MCMC method, whose methodology will hereafter referred to as LTE/MCMC. From the lines of the isotopologues listed in Table 1, we obtained LTE solutions under the main assumption that the ${ }^{13} \mathrm{C} \mathrm{HC}_{3} \mathrm{~N}$ isotopologues and $\mathrm{HC}_{3} \mathrm{~N}$ have the same origin, (i.e. they are confined in a region of up to $6 \operatorname{arcsec}$ and with $T_{\text {exc }}$ between $\sim 80 \mathrm{~K}$ and $90 \mathrm{~K}$ ). The LTE/MCMC models were computed including as free parameters only the column density and excitation temperature. As an upper limit, based on the premise that $\mathrm{HC}_{3} \mathrm{~N}$ must be more abundant than its isotopologues, the ${ }^{13} \mathrm{C}$ column densities were integrated with the condition that $N<N\left(\mathrm{HC}_{3} \mathrm{~N}\right) \approx 1 \times 10^{15} \mathrm{~cm}^{-2}$. A single LTE/MCMC computation was carried out for all the ${ }^{13} \mathrm{C}$ isotopologues to obtain a single excitation solution for all of them. Such computation included all the observed lines of the ${ }^{13} \mathrm{C}$ isotopologues (Table 1 ). We found that the best model $\left(\chi_{\text {red }}^{2}=5.88\right)$ to reproduce the observed lines of $\mathrm{H}^{13} \mathrm{CCCN}, \mathrm{HC}^{13} \mathrm{CCN}$ and $\mathrm{HCC}^{13} \mathrm{CN}$ is the one that assumes $N=(3.3 \pm 0.2) \times 10^{13} \mathrm{~cm}^{-2}$ and $T_{\text {exc }}=82 \pm 5 \mathrm{~K}$ for a source size of 6 arcsec. The LTE/MCMC results are shown in Fig 7 as a corner diagram, where the highest probability distribution of $T_{\text {exc }}$ against $N$ is exhibited. A second approach was made by adopting $T=85 \mathrm{~K}$ (see Sect. 4.2.1) and computing only the column density as a free parameter. The best model $\left(\chi_{\text {red }}^{2}=5.87\right)$ yielded $N=(3.18 \pm 0.04) \times 10^{13} \mathrm{~cm}^{-2}$, which is lower than the column density reported above only by a very small percentage, namely $\sim 4$ percent.

\section{DISCUSSION}

\subsection{Excitation for the $v=0$ and $v 7=1$ states}

In Sects. 4.2.1 and 4.2.2, we used LTE and non-LTE approaches to derive the density and temperature in G331. Obtained values for $T_{\mathrm{k}}$ and $N\left(\mathrm{HC}_{3} \mathrm{~N}\right)$ from both methods are in good agreement for the $v=0$ lines which suggests that detected transitions are almost thermalized $\left(T_{\text {exc }}=T_{\text {kin }}\right)$. However, it is expected that low-energy transitions may sample the emission from both hot and cold gas components, while highly excited transitions are expected to be rather originated from hot gas environments. Thus, high rotational transitions may also be excited by radiative process, which become more efficient as the angular momentum of the upper state increases (high- $J$ levels).

The critical density of a rotational transition $J \rightarrow J-1$ can be estimated as

$$
n_{\mathrm{c}}^{J, J-1} \approx \frac{A_{(J, J-1)}}{\gamma(J, J-1)}
$$

-2 being $\gamma_{(J, J-1)}$ the collisional coefficient for the $J \rightarrow J-1$ transition, and $A_{(J, J-1)}$ its Einstein's transition probability. If $n\left(\mathrm{H}_{2}\right)<<n_{\mathrm{c}}^{J, J-1}$ the excitation temperature is mostly determined by the nearby radiation field instead of the gas kinetic temperature, which implies that the $J$ level is not thermalized $\left(T_{\text {exc }}<T_{\text {kin }}\right)$. Using the exclusive collisional rate coefficients discussed in Faure et al. (2016), we derived critical densities for the $J=38 \rightarrow 37$ transition for temperatures between $80 \mathrm{~K}$ and $100 \mathrm{~K}$ and we obtained values between $1.6 \times 10^{7} \mathrm{~cm}^{-3}$ and $1.3 \times 10^{7} \mathrm{~cm}^{-3}$. These values are a bit lower than that estimated in Sect. 4.2.2 $\left(\sim 2 \times 10^{7}\right.$ $\mathrm{cm}^{-3}$ ), which very likely confirms that the gas density in G331 is high enough for thermalizing the molecule, even at the higher $J$ levels detected in our observations.

The detection of vibrationally excited lines $\left(v_{7}=1\right)$ suggests that the excitation of the molecule might be strongly influenced, besides collisions, by radiative processes. As previously done in the above paragraph, we must compare the ambient density derived in Sect. 4.2.2, with the critical density for the vibrational bending mode $v_{7}=1$. The latter was estimated by Wyrowski et al. (1999), who derived $n_{\mathrm{c}}$ for the $v_{7}=1-0$ transition of $\mathrm{HC}_{3} \mathrm{~N}$ using the far-IR absorption measurements of Uyemura \& Maeda (1974) to estimate the Einstein's transition probability $\left(A_{u, l}\right)$, and using semiempirical formula for vibrational relaxation times as a function of temperature, reduced mass, and energy vibration (Goldsmith et al. 1982), to estimate the collisional coefficient. Then, the authors derived $n_{\mathrm{c}}=4 \times 10^{8} \mathrm{~cm}^{-3}$ for $T=300 \mathrm{~K}$ (a suitable temperature for a hot core). This value is almost one order of magnitude higher than the ambient density derived for G331 in this work. This implies that IR radiative excitation processes (likely originated by the thermal emission of dust grains) are probably more effi- 
cient than collisions in exciting the vibrationally upper state $v_{7}=1$ of the molecule.

In case of IR radiative excitation and assuming that the gas and dust are thermally coupled (a good approximation considering a gas density of $2 \times 10^{7} \mathrm{~cm}^{-3}$; see Sect. 4.2.2) the temperature $T_{\mathrm{vib}}$ determined from the $\mathrm{HC}_{3} \mathrm{~N}$ emission is a good estimate of the dust temperature. Then, we can estimate the IR bolometric luminosity of the hot core by using the Stefan-Boltzmann law (e.g. de Vicente et al. 2000)

$$
\frac{L_{\mathrm{hc}}}{L_{\odot}}=1.8625 \times 10^{-5}\left(\frac{r}{10^{16} \mathrm{~cm}}\right)^{2} T_{\mathrm{dust}}^{4}
$$

were $r$ is the radius of the hot core, and $T_{\text {dust }}$ is the dust temperature. Adopting an upper limit emission size of 6 $\operatorname{arcsec}(\sim 0.22 \mathrm{pc}$ at a distance of $7.5 \mathrm{kpc})$ and a temperature $T_{\text {dust }}=130 \mathrm{~K}$ (see Sect. 4.2.1) we obtained for G331 an IR bolometric luminosity $L \approx 6 \times 10^{6} L \odot$. This value would correspond to a ZAMS star type earlier than O4 (Panagia 1973). In order to test this, we estimated the number of ionizing Lyman continuum photons needed to sustain the ionization in G331 using

$$
N_{\text {Lyc }}=4.5 \times 10^{48} T_{e}^{-0.45} S_{(4800 \mathrm{MHz})} d^{2}
$$

(Simpson \& Rubin 1990) were $d$ is the distance in kpc, $T_{e}$ is the electron temperature (assumed as $10^{4} \mathrm{~K}$ ), and $S_{(4800 \mathrm{MHz})}$ is the radio continuum flux at $4800 \mathrm{MHz}$ in $\mathrm{Jy}$ (adopted as $0.095 \mathrm{Jy}$; Merello et al. 2013b). Keeping in mind that more than 50 percent of the UV photons can be absorbed by the ISM (Inoue 2001), the total amount of ionizing photons needed to sustain the current level of ionization in G331 is $N_{\text {Lyc }} \approx 1 \times 10^{48} \mathrm{~s}^{-1}$. Adopting fluxes extracted from Panagia (1973), we estimate the spectral type of the ionizing source to be about O9/O8.5 (ZAMS). Then, the spectral type of the internal stellar source derived by this method is different than that derived from the IR bolometric luminosity. It should be noted, however, that the latter magnitude is highly dependant on the size of the hot core (see Eq. 12), which for the case of G331 is highly uncertain due to the presence of a powerful outflow.

\section{$5.212 \mathrm{C} / 13 \mathrm{C}$ ratio}

\subsubsection{Isotopic fractionation and astrochemical implications}

As can be seen from Fig. 3, the $J=24-23$ and $J=25-24$ transition lines of the $\mathrm{HCC}^{13} \mathrm{CN}$ isotopologue are brighter than those of the $\mathrm{HC}^{13} \mathrm{CCN}$. This trend can be confirmed from their integrated intensities $\left(\int T_{\mathrm{mb}} d \mathrm{v}\right.$; see Table1). The derived ratio $\mathrm{HCC}^{13} \mathrm{CN} / \mathrm{HC}^{13} \mathrm{CCN}$ of the integrated intensities for the $J=24-23$ line is $1.8 \pm 0.5$, while for the $J=25-24$ line is $1.3 \pm 0.4$. On the other hand, the $J=27-26$ lines for the $\mathrm{H}^{13} \mathrm{CCCN}$ and $\mathrm{HC}^{13} \mathrm{CCN}$ isotopologues have about the same peak temperature (within the errors), a tendency that can also be confirmed by the ratio $\mathrm{H}^{13} \mathrm{CCCN} / \mathrm{HC}^{13} \mathrm{CCN}$ integrated intensities, derived to be $0.9 \pm 0.3$. Then, observed integrated intensities of the $\mathrm{HCC}^{13} \mathrm{CN}$ lines are stronger by a factor between 1.8 to 1.3 than those of $\mathrm{HC}^{13} \mathrm{CCN}$ lines, while there is no significant difference in intensities between $\mathrm{H}^{13} \mathrm{CCCN}$ and $\mathrm{HC}^{13} \mathrm{CCN}$ lines. This kind of anomaly, known as isotopic fractionation, has been reported and studied, not only in $\mathrm{HC}_{3} \mathrm{~N}$ but also in $\mathrm{HC}_{5} \mathrm{~N}$ and other carbon-chain molecules, in several hot and cold sources (e.g. Takano et al. 1998; Sakai et al. 2007, 2013; Li et al. 2016; Araki et al. 2016; Taniguchi et al. 2016a,b, 2017).

The relative abundances ratios of the ${ }^{13} \mathrm{C}$ isotopologues can be also calculated using column densities estimated from the most conspicuous transitions assuming LTE conditions (see Table 2). Thus, the abundance ratios were obtained to be $\left[\mathrm{HCC}^{13} \mathrm{CN}\right]:\left[\mathrm{HC}^{13} \mathrm{CCN}\right]:\left[\mathrm{H}^{13} \mathrm{CCCN}\right]=$ $1.8( \pm 0.5): 1.0: 1.0( \pm 0.4)$. These results seem to confirm that the abundance of $\mathrm{H}^{13} \mathrm{CCCN}$ and $\mathrm{HC}^{13} \mathrm{CCN}$ are comparable with each other, while $\mathrm{HCC}^{13} \mathrm{CN}$ is more abundant than the others. Similar abundance ratios of $\mathrm{HC}_{3} \mathrm{~N}$ were reported in several star-forming and starless cores in the Galaxy (L1527: Taniguchi et al. 2016b; Araki et al. 2016; TMC-1: Takano et al. 1998; G28.28-0.36: Taniguchi et al. 2016b; Serpens South 1A: Li et al. 2016)

Since the isotope exchange reactions seem unlikely in the case of $\mathrm{HC}_{3} \mathrm{~N}$ (Takano et al. 1998), it has been proposed that the differences in abundances in ${ }^{13} \mathrm{C}$ isotopologues are rather a consequence of the formation pathway of the molecule. In the case of $\mathrm{HC}_{3} \mathrm{~N}$ it has been proposed that the neutral-neutral reaction

$$
\mathrm{C}_{2} \mathrm{H}_{2}+\mathrm{CN} \rightarrow \mathrm{HC}_{3} \mathrm{~N}+\mathrm{H}
$$

is the most important chemical pathway to produce $\mathrm{HC}_{3} \mathrm{~N}$ in the cold cloud TMC-1 (Herbst \& Leung 1990; Takano et al. 1998). Further, Chapman et al. (2009) demonstrated that this formation pathway also occurs relatively easy at hot core temperatures $(100-200 \mathrm{~K})$. Thus, the chemical source of the ${ }^{13} \mathrm{C}$ atom can be supplied from $\mathrm{C}_{2} \mathrm{H}_{2}$ and/or $\mathrm{CN}$ favoring a given position where the ${ }^{13} \mathrm{C}$ atom could be located into the molecule. A reaction between predominant ${ }^{13} \mathrm{C}$-enriched $\mathrm{CN}$ with less enriched ${ }^{13} \mathrm{C} \mathrm{C}_{2} \mathrm{H}_{2}$ in the environs of $\mathrm{G} 331$ could then explain the observed abundance ratios $\left[\mathrm{HCC}{ }^{13} \mathrm{CN}\right]:\left[\mathrm{HC}^{13} \mathrm{CCN}\right]:\left[\mathrm{H}^{13} \mathrm{CCCN}\right]=\mathrm{a}: \mathrm{b}: \mathrm{c}$, with $\mathrm{a}>\mathrm{b} \approx \mathrm{c}$ (e.g.Taniguchi et al. 2016b; Araki et al. 2016). It is worth noting that a transition of the molecule ${ }^{13} \mathrm{CN}$ at 217.42856 $\mathrm{GHz}$ was identified in our dataset, next to the $\mathrm{HCC}^{13} \mathrm{CN}$ (24-23) transition line (see Fig. 3).

We accept, however, that the $\mathrm{HC}_{3} \mathrm{~N}$ abundance ratios reported before have large errors. Then, it is important to contrast these results with a multitransition study of rotational lines having larger signal-to-noise ratios (presumably at lower $J$ levels). A complete analysis of APEX data at lower frequencies will be carried out soon. This study would allow us, not only to estimate the abundance ratios of $\mathrm{HC}_{3} \mathrm{~N}$ more accurately, but also to detect some transitions of larger cyanopolyynes (e.g. $\mathrm{HC}_{5} \mathrm{~N}$ ) not detected in our dataset (see below).

\subsubsection{Average ${ }^{12} \mathrm{C} /{ }^{13} \mathrm{C}$ ratio}

As shown in Sect. 4.2.3, the average ${ }^{12} \mathrm{C} /{ }^{13} \mathrm{C}$ ratio was estimated from LTE/MCMC calculations by computing all the isotopologue lines in a single run, which yielded to a ratio of $\sim 21$ (see last line in Table 2). Alternatively, the average ${ }^{12} \mathrm{C} /{ }^{13} \mathrm{C}$ ratio of $\mathrm{HC}_{3} \mathrm{~N}$ in $\mathrm{G} 331$ can be also estimated from the column density of each isotopic species as

${ }^{12} \mathrm{C} /{ }^{13} \mathrm{C}_{\left(\mathrm{HC}_{3} \mathrm{~N}\right)}=\frac{3 \times N_{\mathrm{HC}_{3} \mathrm{~N}}}{N_{\mathrm{H}^{13} \mathrm{CCCN}}+N_{\mathrm{HC}^{13} \mathrm{CCN}}+N_{\mathrm{HCC}^{13} \mathrm{CN}}}$ 


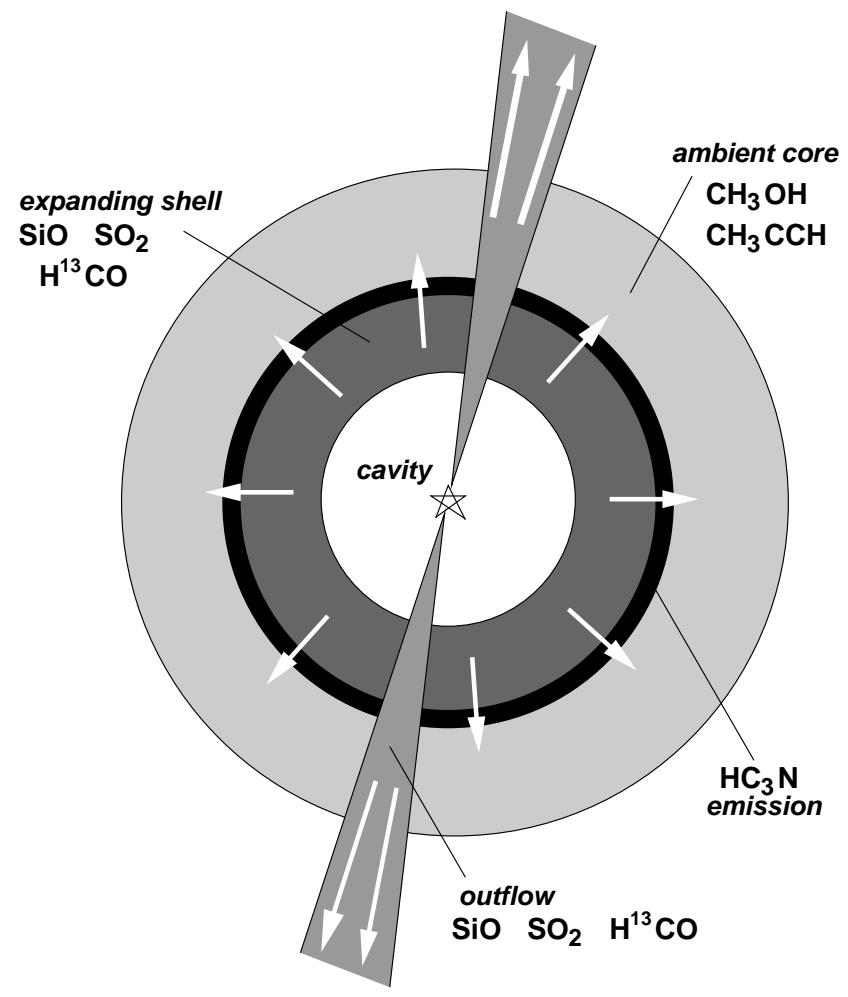

Figure 8. Sketch of the simple model proposed to explain the emission of $\mathrm{HC}_{3} \mathrm{~N}$ and other molecules detected in G331 (based on the models of Merello et al. 2013a and Hervías-Caimapo et al. 2019).

Using Eq. 15 and column densities of each isotopic species indicated in Table 2, the average isotopic ratio was obtained to be $\sim 15$. This result is in relatively good agreement with the ${ }^{12} \mathrm{C} /{ }^{13} \mathrm{C}$ ratios derived from observations of $\mathrm{CH}_{3} \mathrm{OH}$ and $\mathrm{CH}_{3} \mathrm{CN}$ in $\mathrm{G} 331$ (Mendoza et al. 2018b).

In order to compare the ${ }^{12} \mathrm{C} /{ }^{13} \mathrm{C}$ abundance ratio derived for G331 with that expected from its environs (in this case, the giant molecular cloud G331.5-0.1) we used the empirical formula ${ }^{12} \mathrm{C} /{ }^{13} \mathrm{C}=6.21 \times D_{\mathrm{GC}}+18.71$ (derived by Milam et al. 2005 for Galactic GMCs) where $D_{\mathrm{GC}}$ is the distance of G331.5-0.1 to the Galactic center (derived using simple trigonometry). Thus, the expected ${ }^{12} \mathrm{C} /{ }^{13} \mathrm{C}$ abundance ratio for the the molecular cloud G331.5-0.1 is $\sim 43$, more than twice of that derived for G331.

\subsection{The emission of $\mathrm{HC}_{3} \mathrm{~N}$ and larger cyanopolyynes}

As previously mentioned in Sect. 2, high spatial resolution ALMA observations towards G331 revealed the emission of $\mathrm{SiO}(8-7), \mathrm{H}^{13} \mathrm{CO}^{+}(4-3), \mathrm{HCO}^{+}(4-3)$, and $\mathrm{CO}(3-2)$ towards the center of the objects (Merello et al. 2013a). The authors interpreted the emission like a high-velocity jet lying almost along the line of sight with an expanding shocked shell/bubble surrounding the driving source, which is observed in projection as a ring of $\sim 5$ arcsec in size, at the systemic velocity $\left(\sim-90 \mathrm{~km} \mathrm{~s}^{-1}\right)$. The line $J=38-37$ of
$\mathrm{HC}_{3} \mathrm{~N}$ was also reported towards G331 with ALMA observations (Hervías-Caimapo et al. 2019). The authors identified this line as a part of a group of lines $\left(\mathrm{HC}_{3} \mathrm{~N}, \mathrm{SiO}, \mathrm{S}^{18} \mathrm{O}\right.$, $\mathrm{HCO}^{+}$, and $\mathrm{H}^{13} \mathrm{CN}$ ) with broad velocity wings tracing the shocked gas and the outflow, for which a temperature of 160 $200 \mathrm{~K}$ was derived. The second group $\left(\mathrm{CH}_{3} \mathrm{OH}, \mathrm{CH}_{3} \mathrm{CCH}\right.$, and $\left.\mathrm{H}^{13} \mathrm{CO}^{+}\right)$have narrow emission lines and trace a colder $(\sim 70 \mathrm{~K})$ core ambient medium.

In Sect. 4.2, from $\mathrm{HC}_{3} \mathrm{~N}$ lines, we derived temperatures between $\sim 80 \mathrm{~K}$ and $90 \mathrm{~K}$, which are much closer to the temperature of the (quiescent) ambient gas derived by Hervías-Caimapo et al. (2019) than the one of the (expanding) molecular shocked gas. This might indicate that the molecular gas traced by $\mathrm{HC}_{3} \mathrm{~N}$ may be physically associated with molecular gas of the ambient core, which has not been fully disturbed by the plowing power of the expanding shell. This could in turn explain the narrower profile of the $\mathrm{HC}_{3} \mathrm{~N}$ line in the group of lines with broad wings (see fig. 1 from Hervías-Caimapo et al. 2019).

Thus, we can speculate that the emission of $\mathrm{HC}_{3} \mathrm{~N}$ could be originated in a middle region, between the quiescent ambient core and the expanding molecular gas. Then, the molecular gas traced by the $\mathrm{HC}_{3} \mathrm{~N}$ emission might be being disturbed by the expansion of the shell in its inner region, while its undisturbed outer layer preserves much of the physical properties and systemic velocity of the ambient core (see Fig. 8). This is a fairly plausible scenario considering that G331 has shown to be one of the youngest hot cores/outflows known to date. A more detailed analysis of high resolution data is necessary to shed some light on these issues.

Regarding to larger cyanopolyynes, as previously mentioned in Sect. 4, we did not report evidences for $\mathrm{HC}_{5} \mathrm{~N}, \mathrm{HC}_{7} \mathrm{~N}$, nor $\mathrm{HC}_{9} \mathrm{~N}$ (e.g. Sakai et al. 2008; Jaber Al-Edhari et al. 2017; Zhang et al. 2017; Cordiner et al. 2017; Mendoza et al. 2018a; Seo et al. 2019). The absence of $\mathrm{HC}_{5} \mathrm{~N}$ in the current dataset could be associated with several factors, such as (1) insufficient sensitivity in the frequencies of the APEX-1 and APEX-2 data, (2) the excitation regime of the molecular environment, or (3) lower abundances of the molecule. For instance, the second $\mathrm{HC}_{5} \mathrm{~N}(v=0)$ line that should appear in our observations corresponds to the $J=82-81$ transition with $E_{u}=434.8 \mathrm{~K}$ near the transition of $\mathrm{HC}_{3} \mathrm{~N}(v=0)$ appeared at the level $J=24-23$ with $E_{u}=130.98 \mathrm{~K}$ (see Table1). However, in order to conclude on the detection of $\mathrm{HC}_{5} \mathrm{~N}$ in G331 subsequent studies are needed at lower frequencies with instruments such as APEX SEPIA-B5, or with high resolution ALMA Bands 3, 4 and 5. The frequency interval of the mentioned ALMA bands, from $84-211 \mathrm{GHz}$, seems suitable to look for $\mathrm{HC}_{5} \mathrm{~N}$ emission considering the physical and chemical implications of $\mathrm{HC}_{3} \mathrm{~N}$ in G331. For instance, Agúndez et al. (2017) using ALMA data discussed the spatial and chemical correlations between $\mathrm{HC}_{3} \mathrm{~N}$ and $\mathrm{HC}_{5} \mathrm{~N}$ in sources such as IRC+10216.

\section{SUMMARY}

As part of a project aimed at studying the chemical and physical properties prevailing in the massive outflow/hot molecular core G331.512-0.103, we used APEX-1 and APEX-2 observation to detect and analyze the emission 
of the simplest cyanopolyyne molecule $\mathrm{HC}_{3} \mathrm{~N}$, a typical hot core tracer. The main results can be summarized as follows:

(i) We detected thirty-one rotational lines of $\mathrm{HC}_{3} \mathrm{~N}$ above $\sim 3 \mathrm{rms}$ (with the exception of two marginal cases) at $J$ levels between 24 and 39. Seventeen of them correspond to the ground vibrational state $v=0$ (inlcluding 8 lines of ${ }^{13} \mathrm{C}$ isotopologues) and fourteen correspond to the lowest vibrationally excited state $v_{7}=1$.

(ii) From multitransition LTE analyses for the $v=0$ and $v_{7}=1$ beam-diluted lines we estimated excitation temperatures between $\sim 85$ and $89 \mathrm{~K}$, and $\mathrm{HC}_{3} \mathrm{~N}$ column densities between $\sim 6.9 \times 10^{14} \mathrm{~cm}^{-2}$ and $2 \times 10^{15} \mathrm{~cm}^{-2}$. From a non-LTE MCMC/RADEX analysis of the $v=0$ lines, we determined that the emission of $\mathrm{HC}_{3} \mathrm{~N}$ is originated in a region of up to 6 arcsec in size, having an ambient $\mathrm{H}_{2}$ density of $\sim 2 \times 10^{7} \mathrm{~cm}^{-3}$. The results obtained from both, LTE and non-LTE approaches, suggest that detected rotational transitions are almost thermalized $\left(T_{\text {exc }} \approx T_{\text {kin }}\right)$, even at high $J$ levels.

(iii) Using $v=0$ and $v_{7}=1$ lines we determined vibrational temperatures between 130 and $145 \mathrm{~K}$, which are very likely lower limits. The critical density of the $v_{7}=1$ states indicates that IR radiative pumping processes are likely to be more efficient than collisions in exciting the vibrationally upper states of the molecule.

(iv) Using a single-transition LTE analysis, we estimated the column densities of the ${ }^{13} \mathrm{C}$ isotopologues of $\mathrm{HC}_{3} \mathrm{~N}$. Derived values are $(6.7 \pm 0.9) \times 10^{13}$ $\mathrm{cm}^{-2}, \quad(3.8 \pm 0.8) \times 10^{13} \mathrm{~cm}^{-2}$, and $(3.7 \pm 0.6) \times 10^{13}$ $\mathrm{cm}^{-2}$, for $\mathrm{HCC}^{13} \mathrm{CN}, \mathrm{HC}^{13} \mathrm{CCN}$, and $\mathrm{H}^{13} \mathrm{CCCN}$, respectively. Then, derived densities (and integrated intensities of different lines) yields an abundance ratio of about $\left[\mathrm{HCC}^{13} \mathrm{CN}\right]:\left[\mathrm{HC}^{13} \mathrm{CCN}\right]:\left[\mathrm{H}^{13} \mathrm{CCCN}\right]=$ $1.8( \pm 0.5): 1.0: 1.0( \pm 0.4)$. By means of a multi-transition analysis using the LTE/MCMC method, an average solution with $N=(3.3 \pm 0.2) \times 10^{13} \mathrm{~cm}^{-2}$ and $T_{\text {exc }}=82 \pm 5 \mathrm{~K}$ was also determined for the ${ }^{13} \mathrm{C} \quad \mathrm{HC}_{3} \mathrm{~N}$ isotopologues. The ${ }^{12} \mathrm{C} /{ }^{13} \mathrm{C}$ ratios derived from the observations are in agreement with those previously derived in G331 from $\mathrm{CH}_{3} \mathrm{OH}$ and $\mathrm{CH}_{3} \mathrm{CN}$. The evidences for ${ }^{13} \mathrm{C}$ fractionation reported in this work are in line with the widely accepted chemical formation pathway $\mathrm{C}_{2} \mathrm{H}_{2}+\mathrm{CN} \rightarrow \mathrm{HC}_{3} \mathrm{~N}+\mathrm{H}$.

(v) Based on previous ALMA observations and recent results, a very simple physical model was proposed to attempt to shed some light on the emission of $\mathrm{HC}_{3} \mathrm{~N}$ and other molecules previously detected with ALMA.

\section{ACKNOWLEDGEMENTS}

We very much acknowledge the anonymous referee for his/her helpful comments and suggestions that led to the improvement of this paper. This project was partially financed by CONICET of Argentina under projects PIP 00356 and from UNLP, 11/G139. L.B. acknowledges support from CONICYT project Basal AFB-170002.

\section{REFERENCES}

Agúndez M., et al., 2017, A\&A, 601, A4

Araki M., Takano S., Sakai N., Yamamoto S., Oyama T., Kuze N., Tsukiyama K., 2016, ApJ, 833, 291

Belloche A., Müller H. S. P., Menten K. M., Schilke P., Comito C., 2013, A\&A, 559, A47

Bergin E. A., Snell R. L., Goldsmith P. F., 1996, ApJ, 460, 343

Bergner J. B., Guzmán V. G., Öberg K. I., Loomis R. A., Pegues J., 2018, ApJ, 857, 69

Blake G. A., Sutton E. C., Masson C. R., Phillips T. G., 1987, ApJ, 315, 621

Bronfman L., Garay G., Merello M., Mardones D., May J., Brooks K. J., Nyman L.-Å., Güsten R., 2008, ApJ, 672, 391

Chapman J. F., Millar T. J., Wardle M., Burton M. G., Walsh A. J., 2009, MNRAS, 394, 221

Cordiner M. A., Charnley S. B., Kisiel Z., McGuire B. A., Kuan Y.-J., 2017, ApJ, 850, 187

DeLeon R. L., Muenter J. S., 1985, J. Chem. Phys., 82, 1702

Dumke M., Mac-Auliffe F., 2010, in Observatory Operations: Strategies, Processes, and Systems III. p. 77371J, doi: $10.1117 / 12.858020$

Faure A., Lique F., Wiesenfeld L., 2016, MNRAS, 460, 2103

Foreman-Mackey D., Hogg D. W., Lang D., Goodman J., 2013, PASP, 125, 306

Goldsmith P. F., Langer W. D., 1999, ApJ, 517, 209

Goldsmith P. F., Snell R. L., Deguchi S., Krotkov R., Linke R. A., 1982, ApJ, 260, 147

Güsten R., Nyman L. A., Schilke P., Menten K., Cesarsky C., Booth R., 2006, A\&A, 454, L13

Herbst E., Leung C. M., 1990, A\&A, 233, 177

Hervías-Caimapo C., et al., 2019, ApJ, 872, 200

Inoue A. K., 2001, AJ, 122, 1788

Jaber Al-Edhari A., et al., 2017, A\&A, 597, A40

Jiménez-Serra I., Martín-Pintado J., Caselli P., Martín S., Rodríguez-Franco A., Chandler C., Winters J. M., 2009, ApJ, 703, L157

Kim H.-D., Cho S.-H., Chung H.-S., Kim H.-R., Roh D.-G., Kim H.-G., Minh Y. C., Minn Y.-K., 2000, ApJS, 131, 483

Li J., et al., 2016, ApJ, 824, 136

Lovas F. J., Bass J. E., Dragoset R. A., Olsen K. J., 2009, National Institute of Standards and Technology, Gaithersburg, MD

Martín-Pintado J., Jiménez-Serra I., Rodríguez-Franco A., Martín S., Thum C., 2005, ApJ, 628, L61

Mendoza E., et al., 2018a, MNRAS, 475, 5501

Mendoza E., et al., 2018b, ApJ, 853, 152

Merello M., Bronfman L., Garay G., Nyman L.-Å., Evans II N. J., Walmsley C. M., 2013a, ApJ, 774, 38

Merello M., Bronfman L., Garay G., Lo N., Evans II N. J., Nyman L.-A., Cortés J. R., Cunningham M. R., 2013b, ApJ, 774, L7

Milam S. N., Savage C., Brewster M. A., Ziurys L. M., Wyckoff S., 2005, ApJ, 634, 1126

Müller H. S. P., Schlöder F., Stutzki J., Winnewisser G., 2005, Journal of Molecular Structure, 742, 215

Panagia N., 1973, AJ, 78, 929

Peng Y., et al., 2017, ApJ, 837, 49

Pickett H. M., Poynter R. L., Cohen E. A., Delitsky M. L., Pearson J. C., Müller H. S. P., 1998, J. Quant. Spectrosc. Radiative Transfer, 60, 883

Risacher C., et al., 2006, A\&A, 454, L17

Sakai N., Ikeda M., Morita M., Sakai T., Takano S., Osamura Y., Yamamoto S., 2007, ApJ, 663, 1174

Sakai N., Sakai T., Hirota T., Yamamoto S., 2008, ApJ, 672, 371

Sakai N., Takano S., Sakai T., Shiba S., Sumiyoshi Y., Endo Y., Yamamoto S., 2013, Journal of Physical Chemistry A, 117,9831

Sánchez-Monge Á., et al., 2013, A\&A, 552, L10

Seo Y. M., et al., 2019, ApJ, 871, 134 
Simpson J. P., Rubin R. H., 1990, ApJ, 354, 165

Sollins P. K., Megeath S. T., 2004, AJ, 128, 2374

Takano S., et al., 1998, A\&A, 329, 1156

Taniguchi K., Ozeki H., Saito M., Sakai N., Nakamura F., Kameno S., Takano S., Yamamoto S., 2016a, ApJ, 817, 147

Taniguchi K., Saito M., Ozeki H., 2016b, ApJ, 830, 106

Taniguchi K., Ozeki H., Saito M., 2017, ApJ, 846, 46

Taniguchi K., Saito M., Sridharan T. K., Minamidani T., 2018a, ApJ, 854, 133

Taniguchi K., Miyamoto Y., Saito M., Sanhueza P., Shimoikura T., Dobashi K., Nakamura F., Ozeki H., 2018b, ApJ, 866, 32 Taniguchi K., et al., 2018c, ApJ, 866, 150

Uyemura M., Maeda S., 1974, Bull. Chem. Soc. Jpn., 47, 2930

Vassilev V., et al., 2008, A\&A, 490, 1157

Wyrowski F., Schilke P., Walmsley C. M., 1999, A\&A, 341, 882

Zhang X.-Y., Zhu Q.-F., Li J., Chen X., Wang J.-Z., Zhang J.-S., 2017, A\&A, 606, A74

de Vicente P., Martín-Pintado J., Neri R., Colom P., 2000, A\&A, 361, 1058

van der Tak F. F. S., Black J. H., Schöier F. L., Jansen D. J., van Dishoeck E. F., 2007, A\&A, 468, 627

This paper has been typeset from a $\mathrm{T}_{\mathrm{E}} \mathrm{X} / \mathrm{LAT} \mathrm{EX}$ file prepared by the author. 